UNITED STATES DEPARTMENT OF THE INTERIOR

GEOLOGICAL SURVEY

DESCRIPTIONS AND CHEMICAL ANALYSES OF 270 ROCKS AND SOILS
ANALYZED FOR PLATINUM-GROUP METALS
FROM THE IRON CANYON AREA, LANDER COUNTY, NEVADA

By

Robert R. Carlson, 1 Paul E. Venuti, 2

Norman J Page, ${ }^{2}$ and Ted G. Theodore ${ }^{2}$

U.S. Geological Survey

${ }^{1}$ Denver, Colorado 80225

${ }^{2}$ Menlo Park, California 94025

Open-file Report 76-524

1976

This report is preliminary and has

not been edited or reviewed for conformity with Geological Survey

standards and nomenclature 


\title{
DESCRIPTIONS AND CHEMICAL ANALYSES OF 270 ROCKS AND SOILS ANALYZED FOR PLATINUM-GROUP METALS \\ FROM THE IRON CANYON AREA, LANDER COUNTY, NEVADA
}

\section{By}

Robert R. Carlson, 1 Paul E. Venuti, ${ }^{2}$

Norman J Page, ${ }^{2}$ and Ted G. Theodore ${ }^{2}$

\author{
U.S. Geological Survey \\ ${ }^{1}$ Denver, Colorado 80225 \\ ${ }^{2}$ Menlo Park, California 94025
}

\section{Introduction}

The Iron Canyon area is located in the southeastern part of the Antler Peak 15-minute quadrangle, Nevada (fig. 1). This report presents the locations (fig. 2), and descriptions (table 1) and chemical analyses of 270 rocks and soils tested for platinum-group metals from Iron Canyon; 264 are rock samples and six are soil samples. The analyses listed in table 2 are the data used to construct the distribution diagrams and histograms in a report by Theodore and others (1976). Twenty-nine elements ( $\mathrm{Mg}, \mathrm{Fe}, \mathrm{Ca}, \mathrm{Ti}$, As, $\mathrm{Sb}, \mathrm{W}, \mathrm{V}, \mathrm{Mo}, \mathrm{Sn}, \mathrm{Ni}, \mathrm{Cr}, \mathrm{Ba}, \mathrm{Sr}, \mathrm{B}, \mathrm{Pb}, \mathrm{Mn}, \mathrm{Bi}, \mathrm{Be}, \mathrm{Nb}, \mathrm{Y}, \mathrm{Cd}, \mathrm{Cu}, \mathrm{Zr}, \mathrm{Ag}$, $\mathrm{La}, \mathrm{Zn}, \mathrm{Sc}$, and $\mathrm{Co}$ ) were analyzed by a six-step spectrographic method (Grimes and Marranzino, 1968). The spectrographic results are reported to the nearest number in the series $1,0.7,0.5,0.3,0.2,0.15,0.1$, and so on. The precision of a reported value is approximately plus 100 percent or minus 50 percent. Mercury and gold were analyzed by atomic-absorption methods (Vaughn and McCarthy, 1964; Thompson and others, 1968); gold in a few samples was analyzed by fire assay. Platinum, palladium, rhodium, ruthenium, and iridium were analyzed by fire-assay, emission spectrographic techniques (Cooley and others, 1976); rhodium, ruthenium, and iridium were not detected in any of the samples from Iron Canyon at lower detection levels of 0.002 to $0.5 \mathrm{ppm}$. The geology of the Iron Canyon area is described by Roberts (1964), Roberts and Arnold (1965), and Theodore and Roberts (1971).

Spectrographic analyses were made by N. M. Conklin, K. J. Curry, E. L. Mosier, J. M. Motnoka, and K. C. Watts. Platinum-group metal analyses were made by R. R. Carlson, E. F. Cooley, and T. A. Doerge. Gold and mercury analyses were made by W. L. Campbell, L. Dickson, T. G. Ging, R. F. Hansen, W. W. Janes, H. D. King, W. B. Lynch, J. D. Hoffman, C. Huffman, C. L. Jacobson, B. Marshall, E. E. Martinez, P. Martinez, J. D. Menzik, R. L. Miller, J. M. Mitchell, K. R. Murphy, S. L. Noble, M. R. Parker, M. S. Rickard, L. B. Riley, T. A. Roemer, J. A. Thomas, J. E. Troxel, and G. H. VanSickle. 


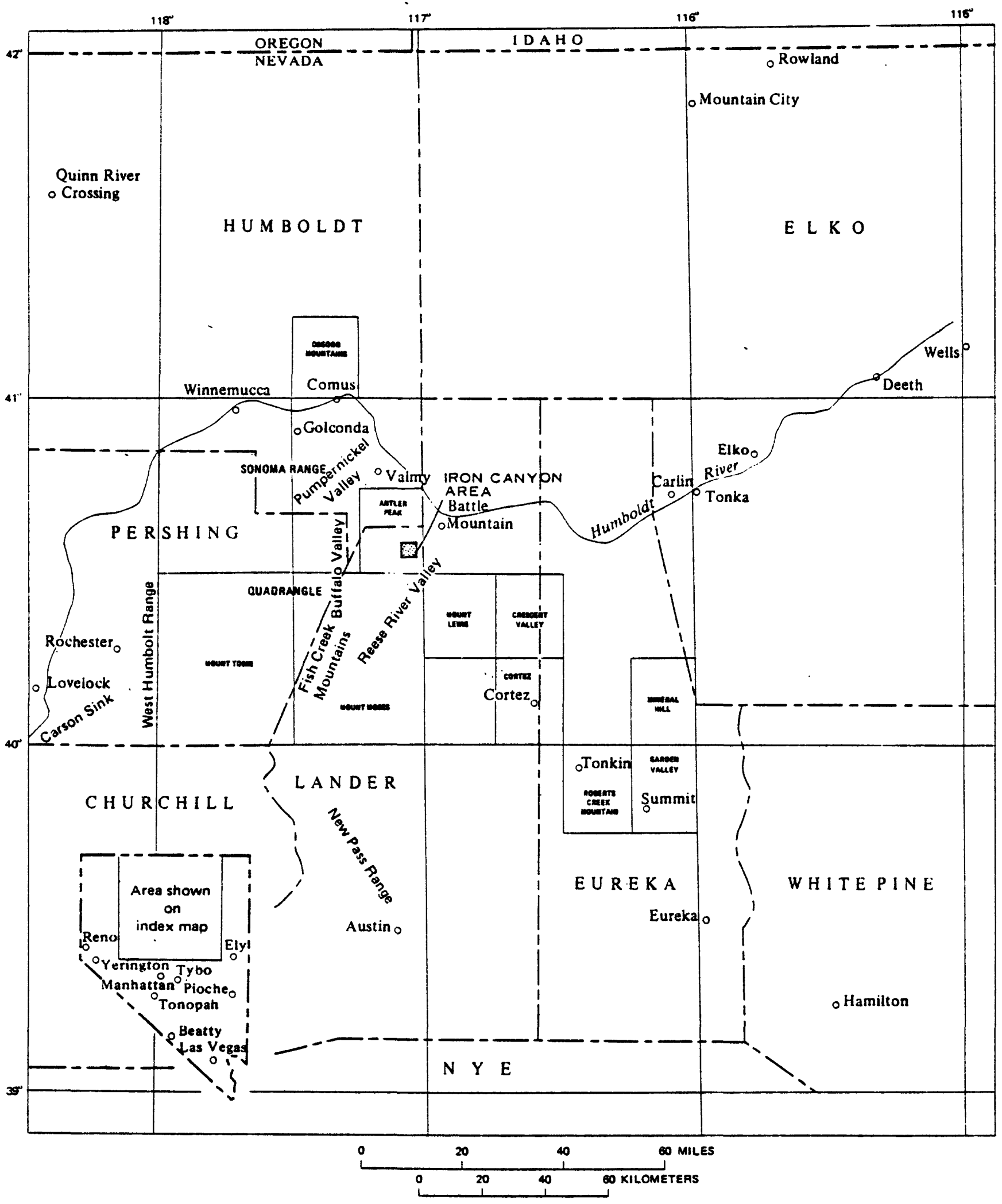

Figuro 1. lowalion map of Lhe Ant ler Peak quadrangle. 


\section{References cited}

Cooley, E. F., Curry, K. J., and Carlson, R. R., 1976, Analysis of the platinum-group metals and gold by fire-assay emission spectrography: Applied Spectroscopy, v. 30, no. 1, p. 52-56.

Grimes, D. J., and Marranzino, A. P., 1968, Direct-current arc and altemating-current spark emission spectrographic field methods for the semiquantitative analysis of geologic materials: U.S. Geol. Survey Circ. 591, 6 p.

Roberts, R. J., 1964, Stratigraphy and structure of the Antler Peak quadrangle, Humboldt and Lander Counties, Nevada: U.S. Geol. Survey Prof. Paper 459-A, P. Al-A93.

Roberts, R. J., and Amold, D. C., 1965, Ore deposits of the Antler Peak quadrangle, Humboldt and Lander Counties, Nevada: U.S. Geol. Survey Prof. Paper 459-B, P. B1-B94.

Theodore, T. G., and Roberts, R. J., 1971, Geochemistry and geology of deep drill holes at Iron Canyon, Lander County, Nevada, with a section on Geophysical logs of drill hole DDH-2 by Charles $\mathrm{J}$. Jablocki: U.S. Geol. Survey Bull. 1318, 32 p.

Theodore, T. G., Venuti, P. E., Page, N. J, and Carlson, R. R., 1976, Maps showing distribution of palladium and other elements in rocks at Iron Canyon, Lander County, Nevada: U.S. Geol. Survey Misc. Field Studies Map MF-790, 2 sheets.

Thompson, C. E., Nakagawa, H. M., and VanSickle, G. H., 1968, Rapid analysis for gold in geologic materials, in Geological Survey research 1968: U.S. Geol. Survey Prof. Paper 600-B, P. B130-B132.

Vaughn, W. W., and McCarthy, J. H., Jr., 1964, An instrumental technique for the determination of submicrogram concentrations of mercury in soils, rocks, and gas, in Geological Survey research 1964: U.S. Geo1. Survey Prof. Paper 501-D, P. D123-D127. 
Table 1.--Sumple descriptions of 270 rocks and soils analyzed from the Iron liunyon area

[SC, Lower or Middle Cambrian Scott Canyon Formation; H, Upper Cambrian Harmony Formation; V, Ordovician

Valmy Formation; $T$, Tertiary quartz monzonite or altered granodiorite porphyry dike]

\begin{tabular}{|c|c|c|}
\hline Sample no. & Formation & Sample description \\
\hline $\begin{array}{r}A D K-095 \\
-113 \\
-130 \\
-136 \\
-147 \\
-152 \\
-166 \\
-174 \\
\text { ADJ }-963 \\
-970\end{array}$ & $\begin{array}{l}-- \\
\overline{-} \\
\overline{\text { SC }} \\
\overline{\mathrm{SC}} \\
\mathrm{SC} \\
\mathrm{SC} \\
\mathrm{sC} \\
\mathrm{SC}\end{array}$ & $\begin{array}{l}\text { Fault breccla } \\
\text { Iron oxide plus vein quartz } \\
\text { Do. } \\
\text { Chert and Iron-oxide-stained argillite } \\
\text { Iron oxide and veln quartz } \\
\text { Chert, sulfide-bearing } \\
\text { Iron oxide and silicifled chert } \\
\text { Chert and shale, Iron-oxide-stained and brecclated } \\
\text { Greenstone, Iron oxide along jolnts } \\
\text { Iron oxide, In chert and shale }\end{array}$ \\
\hline $\begin{array}{r}A D J-981 \\
-987 \\
A B B-844 \\
-853 \\
-876 \\
-883 \\
-888 \\
-908 \\
-916 \\
A D R-951\end{array}$ & $\begin{array}{l}\bar{I} \\
\overline{s c} \\
\overline{-} \\
\overline{s c} \\
\overline{s c}\end{array}$ & $\begin{array}{l}\text { Iron oxide, vein quartz, and chert } \\
\text { Porphyry dike, altered } \\
\text { Chert, Iron-oxide-stalned } \\
\text { Iron oxide and vein quartz } \\
\quad \text { Do. } \\
\text { Iron oxide } \\
\text { Chert, copper-oxide-stained } \\
\text { Iron oxide and vein quartz } \\
\text { Chert Do. }\end{array}$ \\
\hline $\begin{array}{r}\text { ADR- } 957 \\
-973 \\
-976 \\
\text { ADS- } 013 \\
-020 \\
-030 \\
\text { ACO-356 } \\
-359 \\
-419 \\
-456\end{array}$ & $\begin{array}{l}\bar{T} \\
\overline{\text { sc }} \\
\overline{\mathrm{SC}} \\
\mathrm{SC} \\
\mathrm{SC} \\
\mathrm{SC} \\
\mathrm{SC} \\
\mathrm{SC}\end{array}$ & 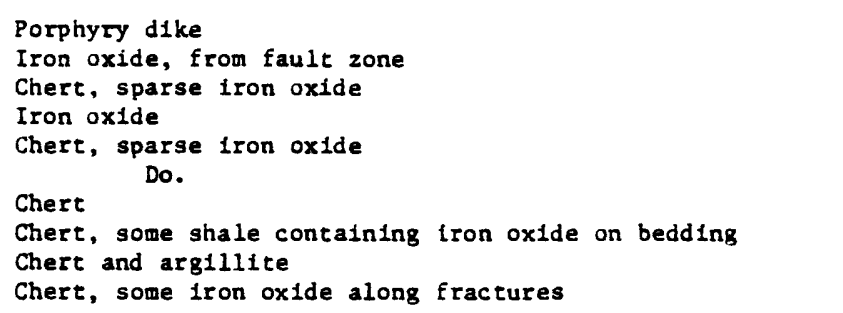 \\
\hline $\begin{array}{r}A C 0-459 \\
-494 \\
-500 \\
-506 \\
-530 \\
-533 \\
-536 \\
-539 \\
-549 \\
-558\end{array}$ & $\begin{array}{l}- \\
\text { SC } \\
\text { SC } \\
\text { SC } \\
\text { SC } \\
\text { B } \\
\text { SC } \\
\text { SC } \\
\text { SC } \\
\text { SC }\end{array}$ & $\begin{array}{l}\text { Iron oxide } \\
\text { Chert, fron-oxide-stained and veined by quartz } \\
\text { Chert and argillite, brecclaced and iron-oxide-stained } \\
\text { Greenstone, altered } \\
\text { Quartzite and chert, abundant iron oxide } \\
\text { Arkose } \\
\text { Greenstone } \\
\text { Chert, some 1ron oxide } \\
\text { Greenstone, abundant fron oxide } \\
\text { Chert, abundant iron oxide }\end{array}$ \\
\hline $\begin{array}{r}\text { ACO-569 } \\
-607 \\
-681 \\
-748 \\
-769 \\
-789 \\
-807 \\
-814 \\
-834 \\
A B H-207\end{array}$ & $\begin{array}{l}\mathrm{H} \\
\mathrm{H} \\
\mathrm{SC} \\
\mathrm{H} \\
\mathrm{H} \\
\mathrm{SC} \\
\mathrm{T} \\
\mathrm{SC} \\
\mathrm{SC} \\
\mathrm{T}\end{array}$ & $\begin{array}{l}\text { Arkose } \\
\text { Arkose, micaceous } \\
\text { Shale } \\
\text { Arkose } \\
\text { Quartzite, pebbly; velned by quartz } \\
\text { Greenstone, sparse Iron oxide } \\
\text { Porphyry, altered and iron-oxide-stained } \\
\text { Chert and Iron oxide } \\
\text { Chert and Iron oxide } \\
\text { Porphyry, altered, and stained by iron oxide }\end{array}$ \\
\hline $\begin{array}{r}\text { ABH-238 } \\
-248 \\
-274 \\
-311 \\
-335 \\
-379 \\
-387 \\
-397 \\
-415 \\
-432\end{array}$ & $\begin{array}{l}\text { SC } \\
\text { H } \\
\text { SC } \\
\text { SC } \\
\text { V } \\
\text { SC } \\
\text { SC } \\
\text { SC } \\
\text { SC }\end{array}$ & $\begin{array}{l}\text { Chert and shale, sparse 1ron oxide } \\
\text { Veln quartz and Iron oxide, trace scorodite } \\
\text { Quartz1te, abundant Iron oxide } \\
\text { Chert } \\
\text { Chert, sparse Iron oxide } \\
\text { Do. } \\
\text { Chert and 1ron oxide } \\
\text { Greenstone, sheared } \\
\text { Chert and vein quartz, moderate iron oxide } \\
\text { Greenstone }\end{array}$ \\
\hline $\begin{array}{r}\text { AEH- } 568 \\
-587 \\
-609 \\
-629 \\
-817 \\
-823 \\
-836 \\
-841 \\
-859 \\
-867\end{array}$ & $\begin{array}{l}\text { SC } \\
\text { SC } \\
\text { SC } \\
\text { SC } \\
\text { SC } \\
\text { SC } \\
\text { SC } \\
\text { SC } \\
\text { SC } \\
\text { - }\end{array}$ & $\begin{array}{l}\text { Chert, Iron oxide, and vein quartz } \\
\text { Chert and iron oxide } \\
\text { Chert and shale, altered } \\
\text { Chert, altered and brecclated; iron oxide along fractures } \\
\text { Chert Do. } \\
\text { Greenstone } \\
\text { Chert, sparse to moderate iron oxide } \\
\text { Chert and Iron oxide } \\
\text { Iron oxide }\end{array}$ \\
\hline
\end{tabular}


Table 1.-Scomple descriptions of 270 rocks and soils conalyzed from the Iron Conyon area-Continued

\begin{tabular}{|c|c|c|}
\hline Sample no. & Formation & Sample description \\
\hline $\begin{array}{r}\text { AEH- }-874 \\
-877 \\
-958 \\
-983 \\
-993 \\
\text { ADR-954 } \\
-955 \\
-960 \\
-968 \\
-969\end{array}$ & $\begin{array}{l}\text { SC } \\
\text { SC } \\
\text { SC } \\
\text { SC } \\
\text { SC } \\
\text { SC } \\
\text { SC } \\
\text { SC } \\
\text { SC }\end{array}$ & $\begin{array}{l}\text { Greenstone } \\
\text { Iron oxide } \\
\text { Chert, sparse iron oxide } \\
\text { Chert and iron oxide } \\
\text { Greenstone } \\
\text { Greenstone, sparse iron oxide } \\
\text { Greenstone, some iron oxide } \\
\text { Greenstone } \\
\text { Greenstone and sparse iron oxide } \\
\text { Do. }\end{array}$ \\
\hline $\begin{array}{r}\text { ADR- } 970 \\
-974 \\
-975 \\
\text { ADK-076 } \\
-077 \\
-078 \\
-079 \\
-080 \\
-081 \\
-082\end{array}$ & $\begin{array}{l}\text { SC } \\
\text { SC } \\
\text { SC } \\
\text { SC } \\
\text { SC } \\
\text { SC } \\
\text { SC } \\
\text { SC } \\
\text { SC } \\
\text { SC }\end{array}$ & $\begin{array}{l}\text { Greenstone } \\
\text { Do. } \\
\text { Do. } \\
\text { Do. } \\
\text { Chert, pod (?) in greenstone } \\
\text { Greenstone, altered } \\
\text { Do. } \\
\text { Do. } \\
\text { Do. } \\
\text { Do. }\end{array}$ \\
\hline $\begin{array}{r}A D K-122 \\
-123 \\
-169 \\
-170 \\
-171 \\
-172 \\
-173 \\
-175 \\
-180 \\
\text { ACO-122 }\end{array}$ & $\begin{array}{l}\text { SC } \\
\text { SC } \\
\text { SC } \\
\text { SC } \\
\text { SC } \\
- \\
\text { SC } \\
\text { SC } \\
\text { SC } \\
\text { SC }\end{array}$ & $\begin{array}{l}\text { Greenstone, Iron-oxide-stained } \\
\text { Do. } \\
\text { Greenstone } \\
\text { Do. } \\
\text { Do. } \\
\text { Calcite } \\
\text { Greenstone } \\
\text { Greenstone and calcite } \\
\text { Greenstone, calcite, and iron oxide } \\
\text { Chert, iron oxide along fractures }\end{array}$ \\
\hline $\begin{array}{r}A C O-123 \\
-124 \\
-125 \\
-126 \\
-127 \\
-128 \\
-129 \\
-130 \\
-131 \\
-132\end{array}$ & $\begin{array}{l}\overline{-} \\
\overline{-} \\
\overline{-} \\
\overline{\text { sc }} \\
\text { SC } \\
\text { SC }\end{array}$ & $\begin{array}{l}\text { Iron oxide, from minor shear zone } \\
\text { Soll sample, collected } 15 \mathrm{~cm} \text { below ground surface } \\
\text { Do. } \\
\text { Do. } \\
\text { Do. } \\
\text { Do. } \\
\text { Do. } \\
\text { Do. } \\
\text { Cheens, iron oxide along bedding }\end{array}$ \\
\hline $\begin{array}{r}\text { ACO-133 } \\
-134 \\
-135 \\
-136 \\
-137 \\
-138 \\
-139 \\
-140 \\
-141 \\
-142\end{array}$ & $\begin{array}{l}\text { SC } \\
\text { SC } \\
\text { SC } \\
-- \\
\text { H } \\
\text { I } \\
-- \\
-- \\
-\end{array}$ & $\begin{array}{l}\text { Chert } \\
\text { Do. } \\
\text { Iron uxide } \\
\text { Quartzite } \\
\text { Porphyry dike } \\
\text { Fault breccia } \\
\text { Do. } \\
\text { Do. } \\
\text { Do. }\end{array}$ \\
\hline $\begin{array}{r}A C O-143 \\
-144 \\
-145 \\
-146 \\
-147 \\
-148 \\
-149 \\
-150 \\
-151 \\
-152\end{array}$ & $\begin{array}{l}-\overline{-} \\
\overline{s c} \\
\text { sc } \\
\text { SC } \\
\text { SC } \\
\overline{\text { SC }} \\
\text { SC }\end{array}$ & $\begin{array}{l}\text { Fault breccia } \\
\text { Do. } \\
\text { Do. } \\
\text { Chert, Iron oxide along bedding } \\
\text { Greenstone } \\
\text { Chert, moderate iron oxide along bedding } \\
\text { Chert, sparse fron oxide along bedding } \\
\text { Iron oxide } \\
\text { Chert, fron oxide stained and veined by quartz } \\
\text { Do. }\end{array}$ \\
\hline $\begin{array}{r}A C 0-153 \\
-154 \\
-155 \\
-156 \\
-157 \\
-158 \\
-159 \\
-160 \\
-161 \\
-162\end{array}$ & $\begin{array}{l}\mathrm{SC} \\
\overline{\mathrm{SC}} \\
\mathrm{T} \\
\mathrm{SC} \\
\mathrm{T} \\
\overline{\mathrm{T}}, \mathrm{SC} \\
\mathrm{T}\end{array}$ & $\begin{array}{l}\text { Greenstone } \\
\text { Soil sample, collected above sample ACO-153 } \\
\text { Greenstone } \\
\text { Porphyry dike } \\
\text { Greenstone } \\
\text { Porphyry dike } \\
\text { Vein quartz } \\
\text { Porphyry and chert, lron-oxide-stained } \\
\text { Porptyry, altered and stained by iron oxide } \\
\text { Porphyry, altered }\end{array}$ \\
\hline
\end{tabular}


Table 1.--Sample descriptions of 270 rocks and soils analyzed from the Iron Canyon area--Continued

\begin{tabular}{|c|c|c|}
\hline Sample no. & Formation & Sample description \\
\hline $\begin{array}{r}A C 0-322 \\
-323 \\
-324 \\
-325 \\
-326 \\
-327 \\
-328 \\
-329 \\
-330 \\
-331\end{array}$ & $\begin{array}{l}\overline{-} \\
\overline{s c} \\
\overline{s c} \\
\bar{z} \\
\overline{-}\end{array}$ & $\begin{array}{l}\text { Iron oxide along Bucte fault, dark-brick-red } \\
\text { Iron oxide } \\
\text { Chert and shale, sheared, iron-oxide-stained } \\
\text { Chert and shale, dark-red iron oxide; some scorodite } \\
\text { Fault gouge, mainly sheared chert and shale } \\
\text { Porphyry, altered } \\
\text { Fault breccla, altered to clay and stained by fron oxide } \\
\text { Porphyry, altered and brecclated } \\
\text { Fault gouge } \\
\text { Do. }\end{array}$ \\
\hline $\begin{array}{r}\text { ACO- } 332 \\
-333 \\
-334 \\
-335 \\
-336 \\
-337 \\
-338 \\
-339 \\
-340 \\
-341\end{array}$ & $\begin{array}{l}-\overline{-} \\
\overline{S C} \\
\overline{S C} \\
\mathrm{~T} \\
\mathrm{SC} \\
\overline{\mathrm{T}} \\
--\end{array}$ & $\begin{array}{l}\text { Do. } \\
\text { Do. } \\
\text { Chert, Iron-oxide-stained and brecclated } \\
\text { Iron oxide, along Butte fault } \\
\text { Chert, brecclated and stained heavily by iron oxide } \\
\text { Porphyry, altered Intensely to clay } \\
\text { Chert, intensely stalned by fron oxide, brecciated } \\
\text { Iron oxide, along Butte fault } \\
\text { Porphyry, altered intensely to clay } \\
\text { Iron oxide, along Butte fault }\end{array}$ \\
\hline $\begin{array}{r}A C 0-342 \\
-343 \\
-344 \\
-345 \\
-346 \\
-367 \\
-368 \\
-369 \\
-370 \\
-374\end{array}$ & $\begin{array}{l}\text { SC } \\
\overline{-} \\
\overline{-} \\
\text { SC } \\
\text { SC } \\
\overline{-}\end{array}$ & $\begin{array}{l}\text { Chert and shale, sheared, altered to clay } \\
\text { Porphyry, altered, stained lntensely by iron oxide } \\
\text { Fault breccla, abundant iron oxide } \\
\text { Iron oxide, along Butte fault } \\
\text { Clay gouge, along Butte fault } \\
\text { Shale, some tron oxide } \\
\text { Chert and tron oxide } \\
\text { Iron oxide, chert, athd argillite } \\
\text { Iron oxide, some scorodite } \\
\text { Iron oxide }\end{array}$ \\
\hline $\begin{array}{r}A C 0-375 \\
-376 \\
-377 \\
-378 \\
-379 \\
-380 \\
-381 \\
-382 \\
-383 \\
-384\end{array}$ & $\begin{array}{l}\text { SC } \\
\text { SC } \\
\text { SC } \\
\mathrm{T} \\
\mathrm{V} \\
-\overline{\mathrm{V}} \\
\mathrm{V} \\
\overline{\mathrm{SC}}\end{array}$ & $\begin{array}{l}\text { Argillite and chert } \\
\text { Argillite } \\
\text { Chert, abundant iron oxtde } \\
\text { Porphyry, altered } \\
\text { Quartzite } \\
\text { Iron oxide, collected } 15 \mathrm{~cm} \text { from fault } \\
\text { Quartzite and pebbly sandstone, altered } \\
\text { Quartzite } \\
\text { Iron oxide } \\
\text { Chert and argillite, some tron oxide }\end{array}$ \\
\hline $\begin{array}{r}A C 0-385 \\
-386 \\
-387 \\
-388 \\
-389 \\
-390 \\
-391 \\
-392 \\
-393 \\
-394\end{array}$ & $\begin{array}{l}\text { SC } \\
\text { SC } \\
\text { SC } \\
\mathrm{T} \\
\mathrm{V} \\
\overline{\mathrm{SC}} \\
\overline{--} \\
\overline{\mathrm{V}}\end{array}$ & $\begin{array}{l}\text { Argillite and chert, some iron oxide } \\
\text { Argillite, stained by iron oxide } \\
\text { Argillite and chert, abundant veln quartz } \\
\text { Porphyry dike, Iron oxide along fractures } \\
\text { Quartzite } \\
\text { Iron oxide } \\
\text { Chert and argillite, stained intensely by iron oxide } \\
\text { Iron oxide } \\
\quad \text { Do. } \\
\text { Quartzice }\end{array}$ \\
\hline $\begin{array}{r}\text { ACO- } 395 \\
-396 \\
-397 \\
-398 \\
-399 \\
-400 \\
-401 \\
-402 \\
-403 \\
-404\end{array}$ & $\begin{array}{l}\mathrm{V} \\
\overline{\mathrm{T}} \\
\overline{\mathrm{V}} \\
\mathrm{SC} \\
\mathrm{SC} \\
\mathrm{SC} \\
\mathrm{SC} \\
\mathrm{SC}\end{array}$ & $\begin{array}{l}\text { Quartzite, tron oxide along fractures } \\
\text { Iron oxide, along alnor fault } \\
\text { Porphyry dike, along fault } \\
\text { Iron oxide, along fault } \\
\text { Quartzite, abundant 1ron oxide } \\
\text { Chert, abundant fron oxide } \\
\text { Chert and argillite } \\
\text { Chert and argillite, stalned by fron oxide } \\
\text { Chert, stained by iron oxide } \\
\text { Chert }\end{array}$ \\
\hline $\begin{array}{r}A C 0-405 \\
-406 \\
-407 \\
-408 \\
-409 \\
-410 \\
-411 \\
-412 \\
-413 \\
-414\end{array}$ & $\begin{array}{l}\overline{S C} \\
\mathrm{~T} \\
\mathrm{SC} \\
\mathrm{SC} \\
\mathrm{SC} \\
\mathrm{SC} \\
\overline{\mathrm{T}} \\
-\end{array}$ & $\begin{array}{l}\text { Iron oxide } \\
\text { Chert, stained by Iron oxide } \\
\text { Porphyry } \\
\text { Chert, stained by Iron oxide } \\
\text { Chert, stained by 1ron oxide and veined by quartz } \\
\text { Chert and argillite } \\
\text { Greenstone } \\
\text { Iron oxide } \\
\text { Porphyry dike } \\
\text { Iron oxide }\end{array}$ \\
\hline
\end{tabular}


Table 1.--Sample descriptions of 270 rocks and soils analyzed from the Iron Canyon area--Continued

\begin{tabular}{|c|c|c|}
\hline Sample no. & Formation & Sample descriptions \\
\hline $\begin{array}{r}A C O-415 \\
A E H-492 \\
-493 \\
-494 \\
-495 \\
-496 \\
-497 \\
-498 \\
-499 \\
-500\end{array}$ & $\begin{array}{l}\text { SC } \\
\text { SC } \\
\text { SC } \\
\text { SC } \\
\text { SC } \\
\text { SC } \\
\text { SC } \\
\text { SC } \\
\text { T } \\
\text { SC }\end{array}$ & $\begin{array}{l}\text { Chert, stained by fron oxide } \\
\text { Greenstone } \\
\text { Do. } \\
\text { Do. } \\
\text { Do. } \\
\text { Do. } \\
\text { Do. } \\
\text { Do. } \\
\text { Porphyry dike, altered } \\
\text { Greenstone }\end{array}$ \\
\hline $\begin{array}{r}\text { AEH-501 } \\
-502 \\
-503 \\
-504 \\
-505 \\
-506 \\
-507 \\
-508 \\
-509 \\
-510\end{array}$ & $\begin{array}{l}\text { SC } \\
\text { SC } \\
\text { SC } \\
\text { T } \\
\text { SC } \\
\text { T } \\
\text { SC } \\
\text { SC } \\
\text { SC } \\
\text { SC }\end{array}$ & $\begin{array}{l}\text { Do. } \\
\text { Do. } \\
\text { Do. } \\
\text { Porphyry } \\
\text { Greenstone } \\
\text { Porphyry } \\
\text { Greenstone } \\
\text { Do. } \\
\text { Do. } \\
\text { Chert, sparse 1ron oxide }\end{array}$ \\
\hline $\begin{array}{r}\text { AEH- } 511 \\
-512 \\
-514 \\
-515 \\
-517 \\
-520 \\
-521 \\
-522 \\
-523 \\
-524\end{array}$ & $\begin{array}{l}\text { SC } \\
\text { SC } \\
\overline{S C} \\
- \\
\overline{-} \\
\overline{S C} \\
\text { SC }\end{array}$ & $\begin{array}{l}\text { Chert, fron oxide along fracture } \\
\text { Greenstone } \\
\text { Do. } \\
\text { Iron oxide } \\
\text { Greenstone, some Iron oxide } \\
\text { Iron oxide } \\
\text { Do. } \\
\text { Vein quartz and sulfides } \\
\text { Chert, altered, stained by Iron oxide } \\
\text { Do. }\end{array}$ \\
\hline $\begin{array}{r}\text { AEH- } 525 \\
-526 \\
-527 \\
-528 \\
-529 \\
-531 \\
-532 \\
-533 \\
-534 \\
-535\end{array}$ & $\begin{array}{l}\text { SC } \\
\text { SC } \\
\text { SC } \\
\text { SC } \\
\text { SC } \\
\text { SC } \\
- \\
\text { I } \\
\text { SC }\end{array}$ & $\begin{array}{l}\text { Chert and 1ron oxide } \\
\text { Do. } \\
\text { Greenstone } \\
\text { Do. } \\
\text { Do. } \\
\text { Greenstone, altered and stained by 1ron oxide } \\
\text { Iron oxide } \\
\text { Porphy dike, altered; some Iron oxide } \\
\text { Chert } \\
\text { Greenstone }\end{array}$ \\
\hline $\begin{array}{l}\text { AEH- } 536 \\
-537 \\
-538 \\
-594 \\
-595 \\
-596 \\
-597 \\
-598 \\
-599 \\
-600\end{array}$ & $\begin{array}{l}\text { SC } \\
\text { SC } \\
\text { SC } \\
\overline{\text { sc }} \\
\overline{\text { SC }} \\
\mathrm{SC}\end{array}$ & $\begin{array}{l}\text { Chert and 1ron oxide } \\
\text { Do. } \\
\text { Do. } \\
\text { Chert and shale, sparse iron oxide } \\
\text { Iron oxide } \\
\text { Greenstone, altered, and stained by Iron oxide } \\
\text { Iron oxide } \\
\text { Greenstone } \\
\text { Greenstone, altered, and stained by fron oxide } \\
\text { Iron oxide }\end{array}$ \\
\hline $\begin{array}{r}\text { AEH- }-601 \\
-602 \\
-603 \\
-656 \\
-657 \\
-658 \\
-659 \\
-660 \\
-661 \\
-662\end{array}$ & $\begin{array}{l}\text { T } \\
\text { SC } \\
\text { SC } \\
\text { SC } \\
\text { SC } \\
\text { SC } \\
\text { SC } \\
\text { SC } \\
\text { SC } \\
\text { SC }\end{array}$ & $\begin{array}{l}\text { Porphyry, altered } \\
\text { Greenstone } \\
\text { Do. } \\
\text { Chert, sparse Iron oxide } \\
\text { Do. } \\
\text { Greenstone } \\
\text { Do. } \\
\text { Greenstone and fron oxide } \\
\text { Greenstone and sparse Iron oxide } \\
\text { Do. }\end{array}$ \\
\hline
\end{tabular}


Table 2.--Semiquantitative spectrographic analyses and chemical analyses for $\mathrm{Au}, \mathrm{Hg}, \mathrm{Pt}$, and $\mathrm{Pd}$ of 270 rocks and soils from the Iron Canyon area

[G, greater than upper limit of determination or greater than value shown; $N$, not detected at limit of detection or at value shown; $L$, detected but below limit of determination or below value shown; $B$, not determined. Analysts: Spectrographic results, N. M. Conklin, K. J. Curry, E. L. Mosier, J. M. Motooka, K. C. Watts; Pt-group metals, R. R. Carlson, E. F. Cooley, T. A. Doerge; Au and Hg, W. L. Campbell, L. Dickson, T. G. Ging, R. F. Hansen, W. W. Janes, H. D. King, W. B. Lynch, J. D. Hoffman, C. Huffman, C. L. Jacobson, B. Marsha11, E. E. Martinez, P. Martinez; J. D. Menzik, R. L. Miller, J. M. Mitchell, K. R. Murphy, S. L. Noble, M. R. Parker, M. S. Rickard, L. B. Riley,

T. A. Roemer, J. A. Thomas, J. E. Troxel, and G. H. VanSickle]

(tabular material follows) 


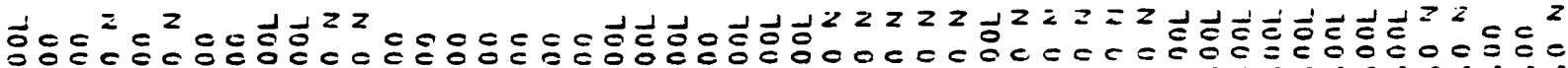

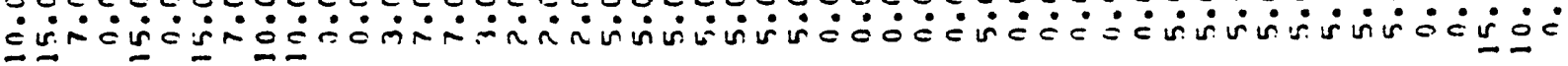

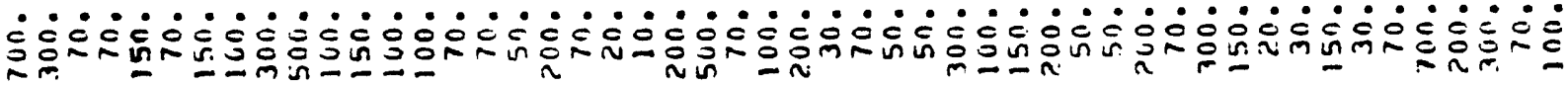

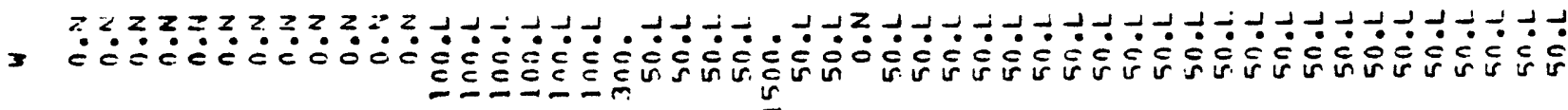

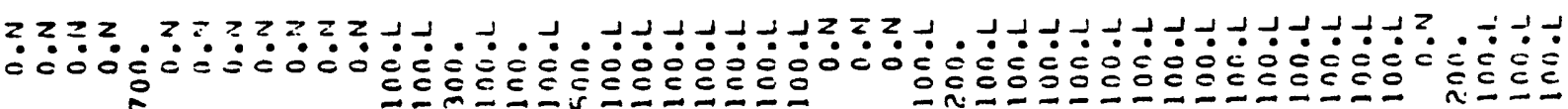

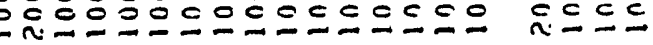

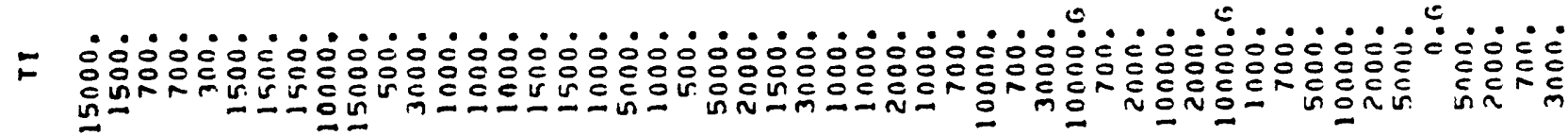

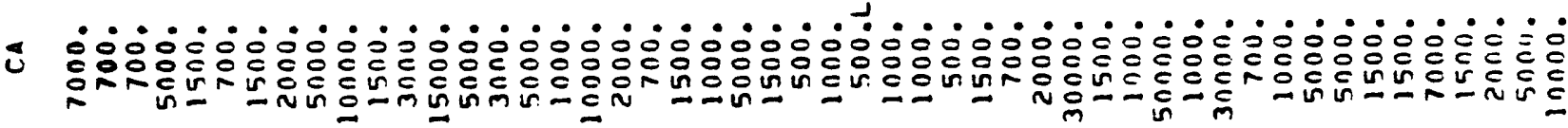

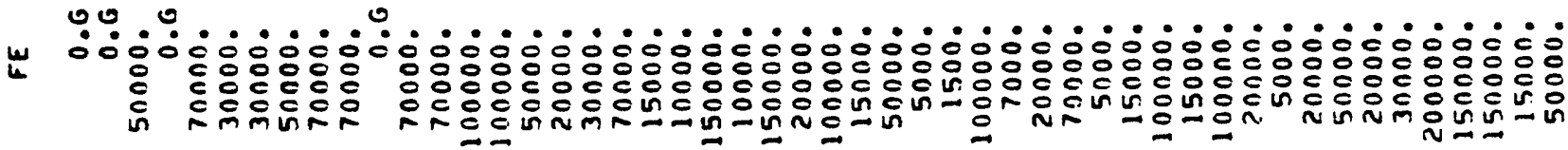

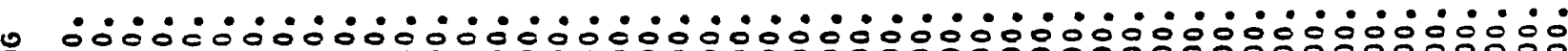

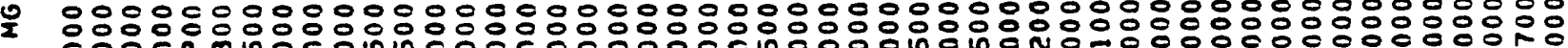

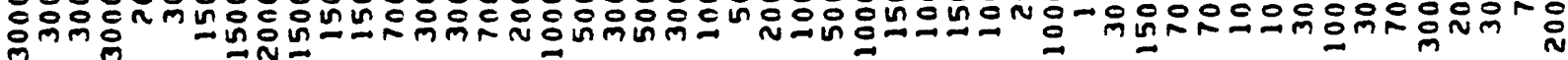


z

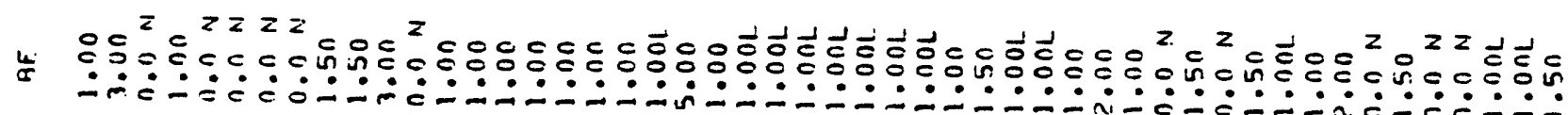

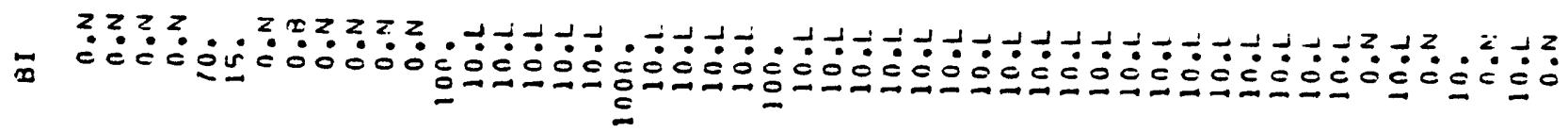

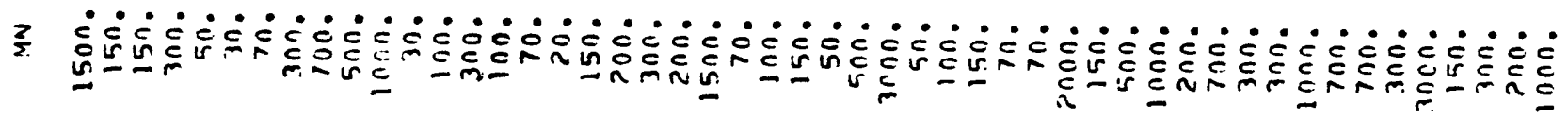

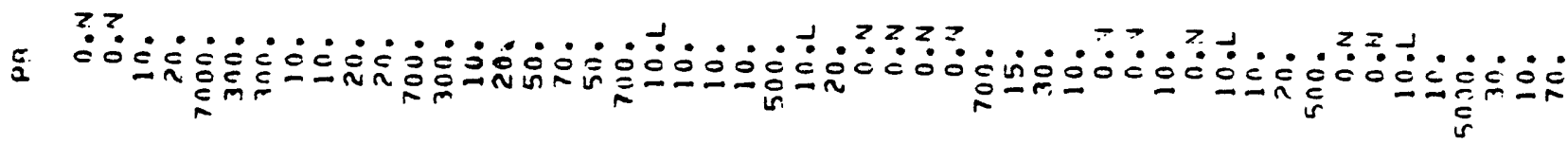

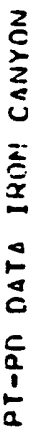

a

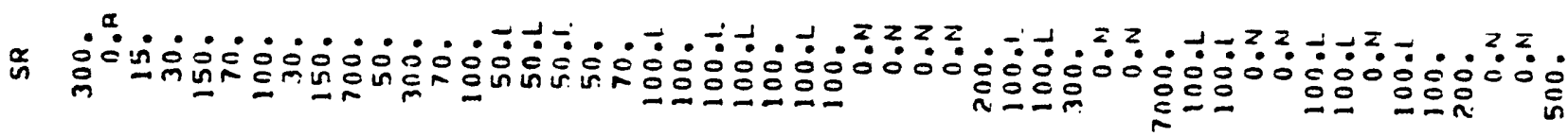

- $\dot{0} \dot{0} \dot{0} \dot{0} \dot{0} \dot{0} \dot{0} \dot{0} \dot{0} \dot{0} \dot{0} . . . . . . .$.

mín

( ñ⿺辶一்

z

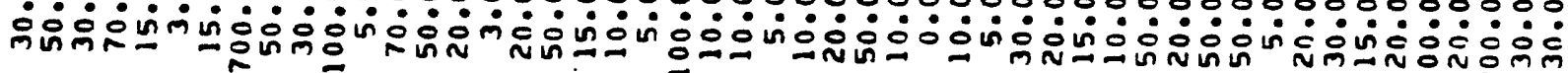




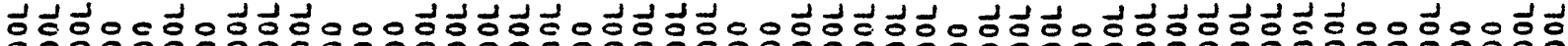

द

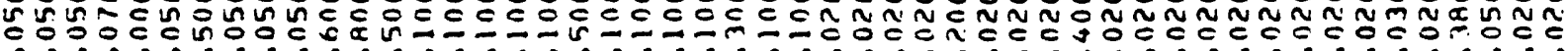

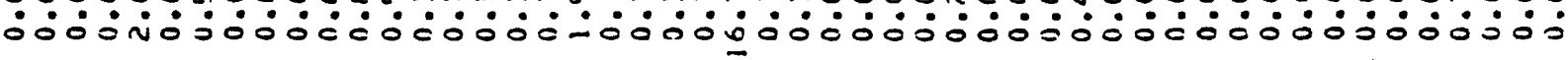

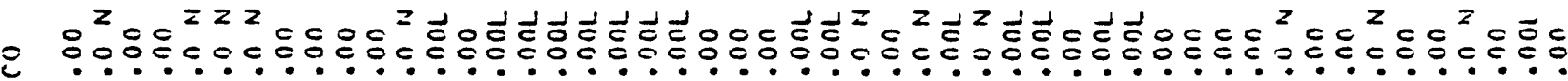

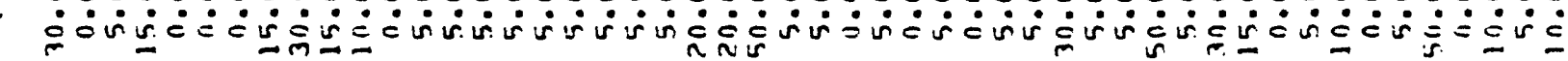

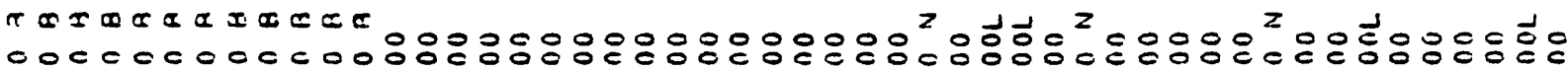

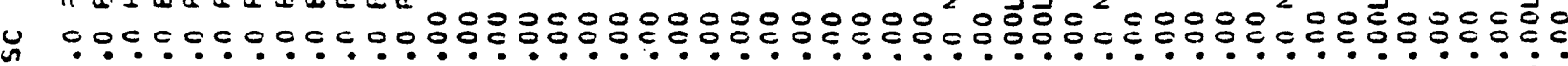

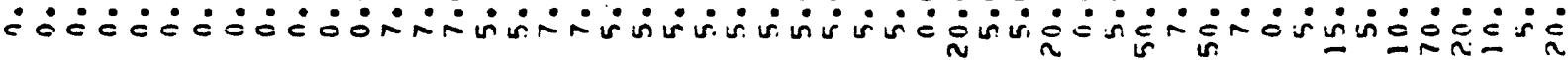

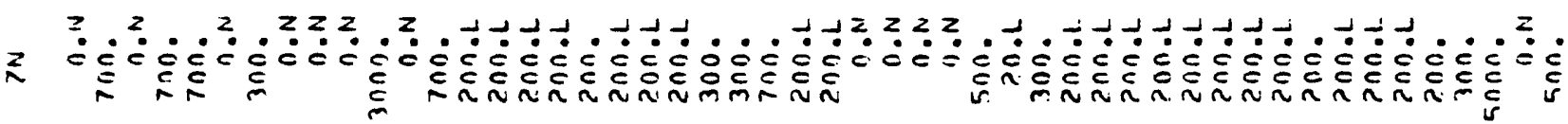

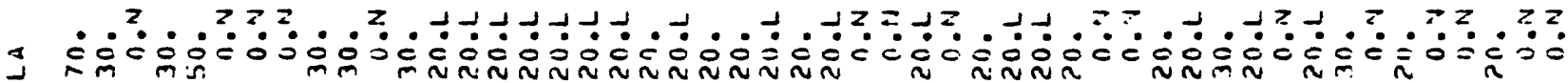

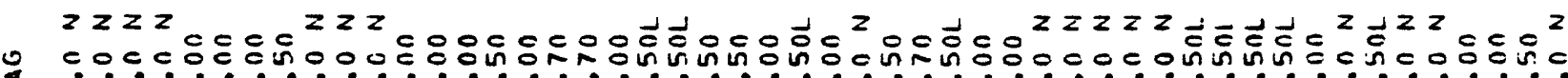

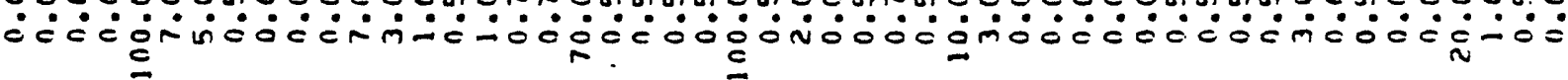

$a \propto \alpha \propto \alpha x \propto ⿻ x \pi$

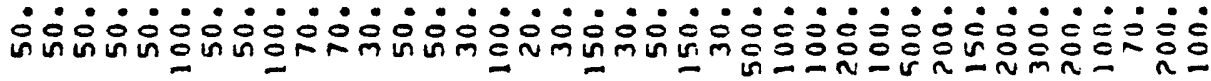

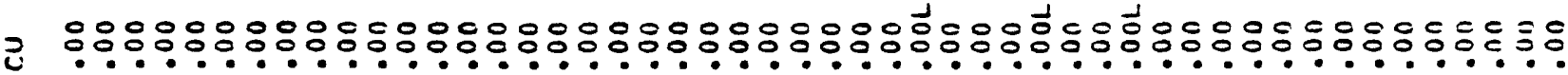

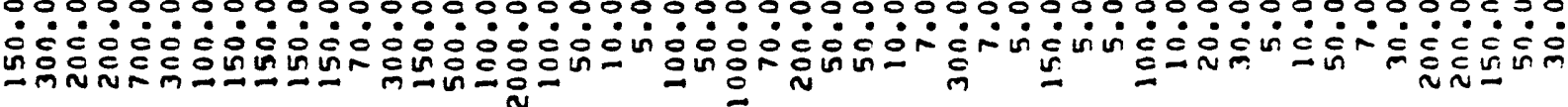

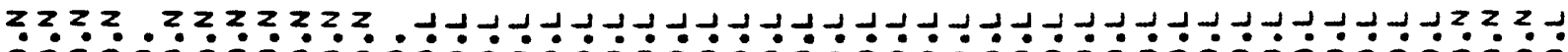

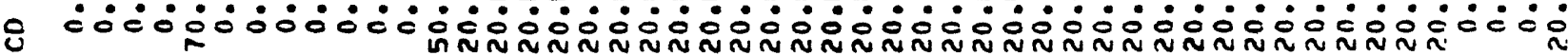

>

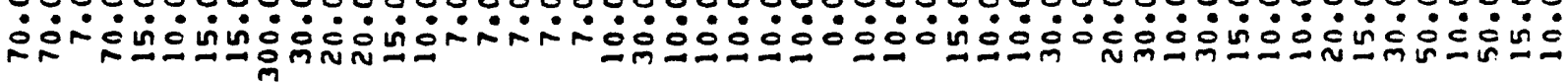




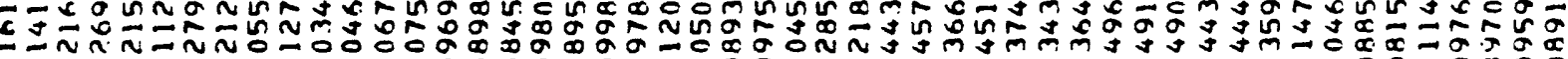

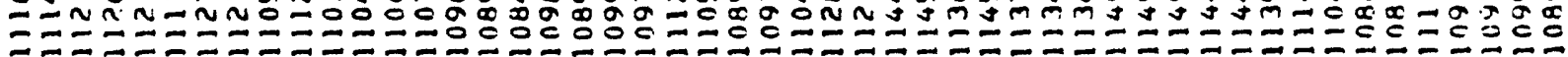

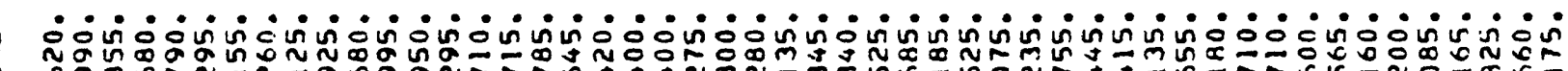

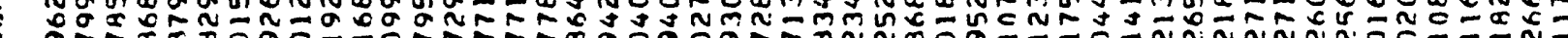

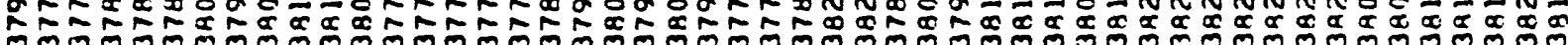

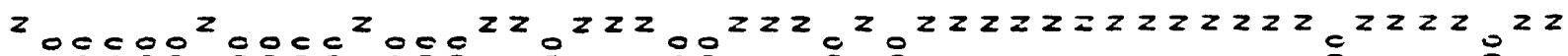
을

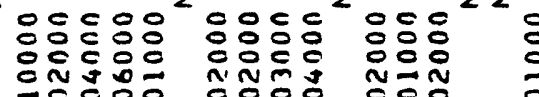

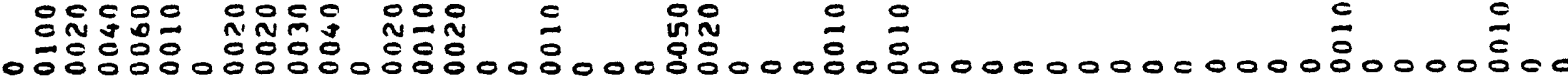

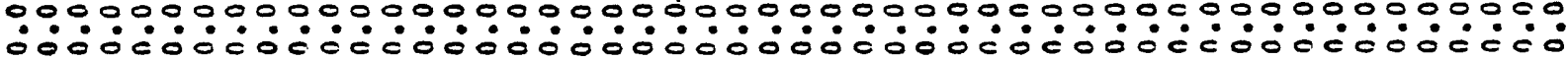

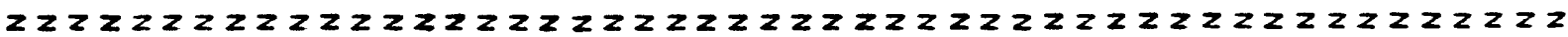
a.

00000000000000000000000000000000000000000000000000

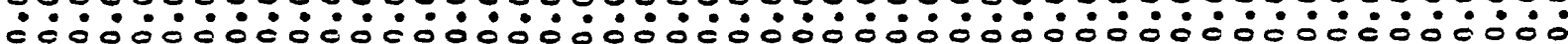

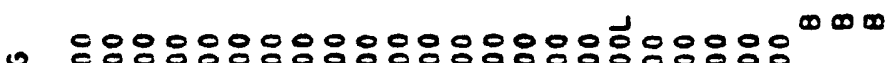

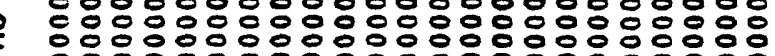

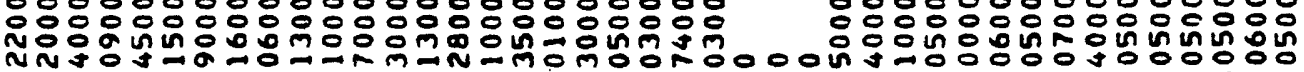

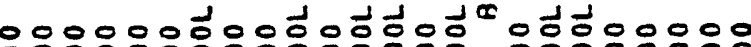

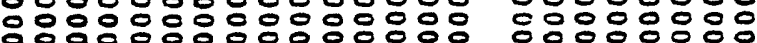

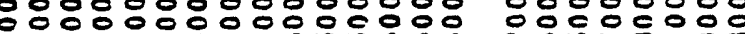

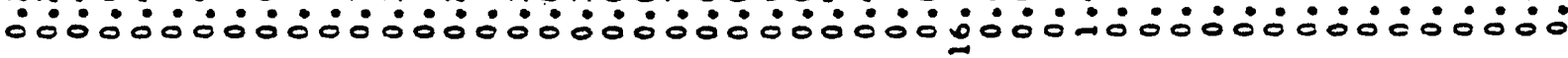




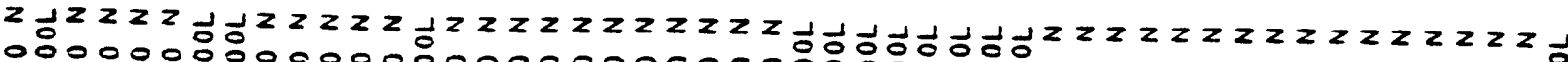

I $00900000000000000000000000000000000=00000000=000000$

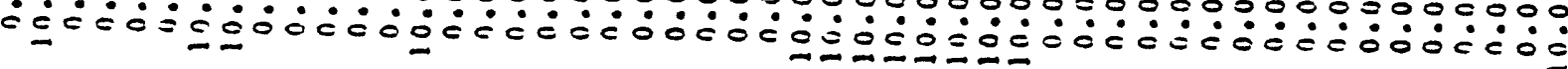

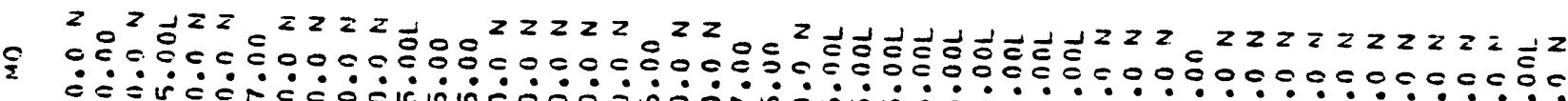

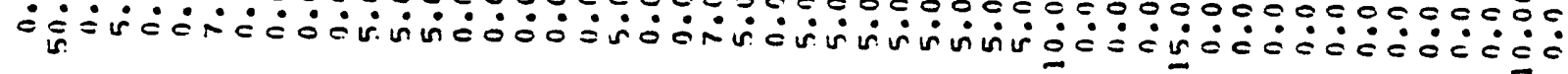

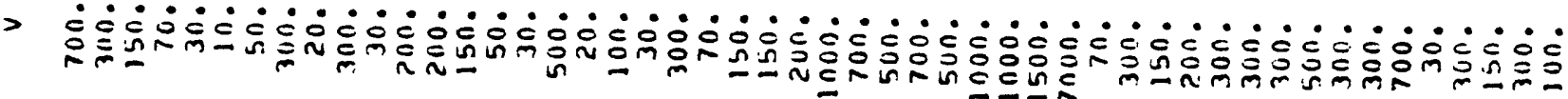

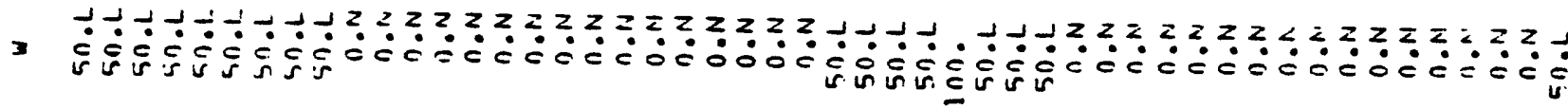

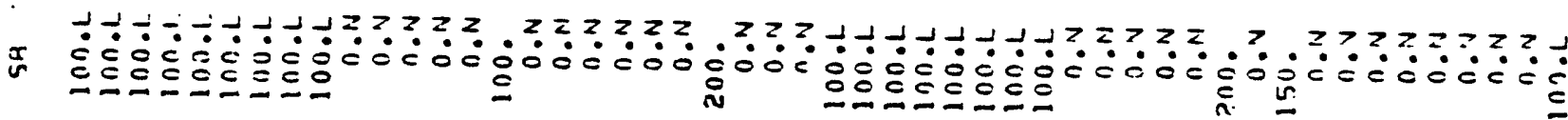

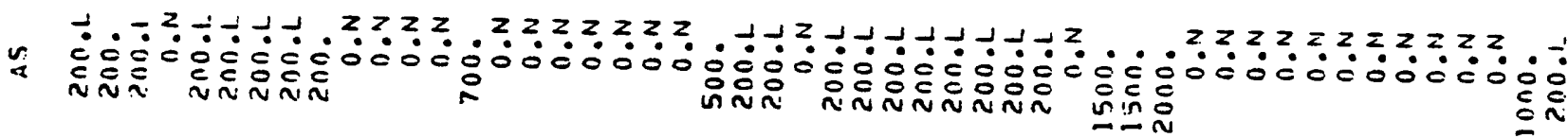

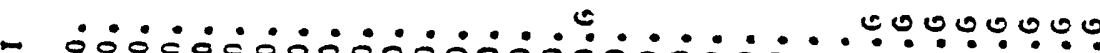

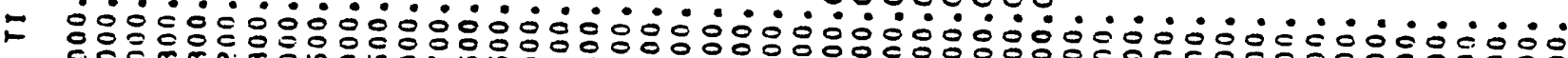

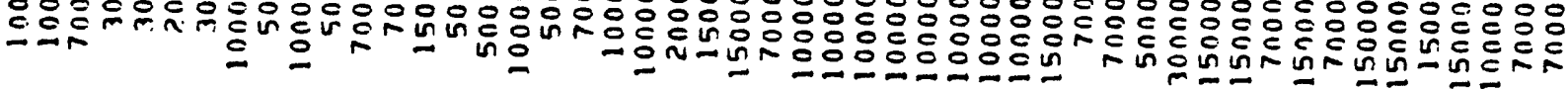

$\frac{1}{a}$

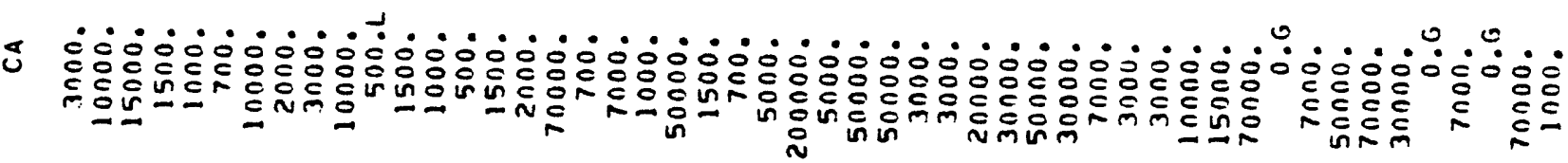
u

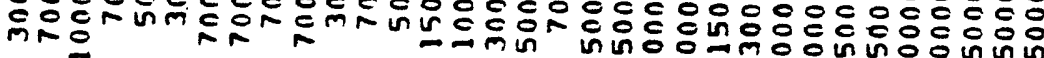

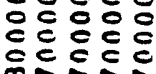

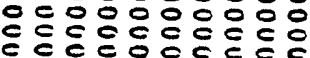

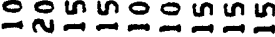

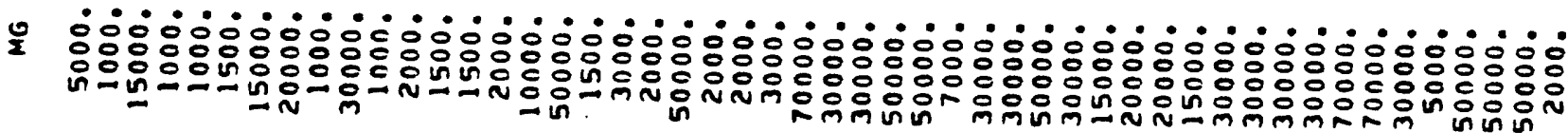

\footnotetext{
แ

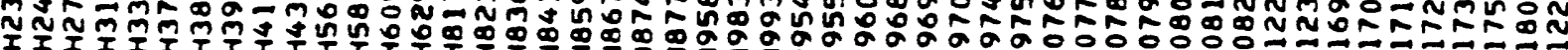

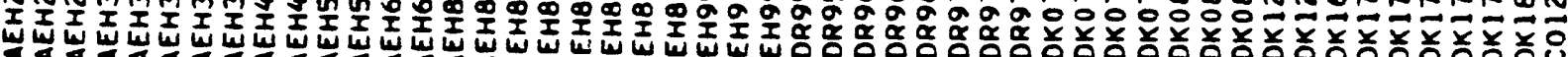
i $\psi$
} 
F

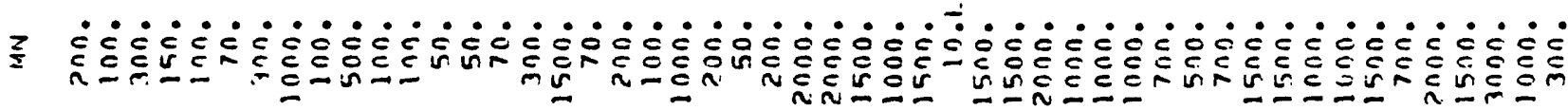

a

๔

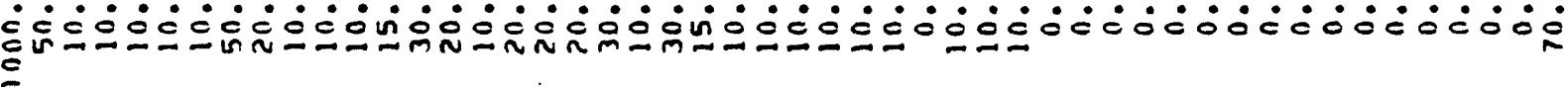

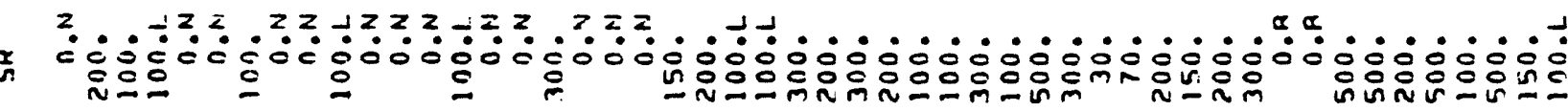

×

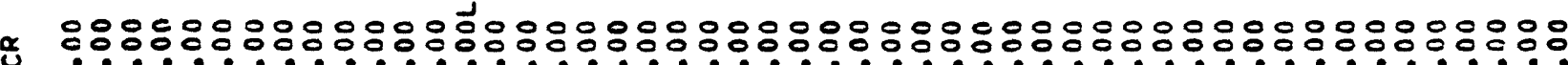

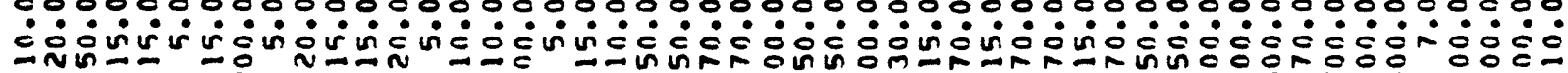
ㅇ

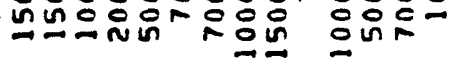

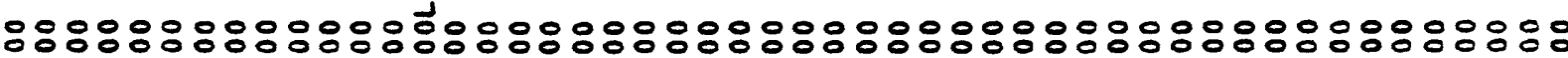

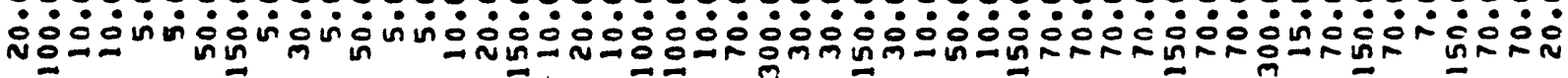




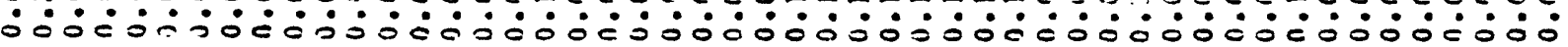

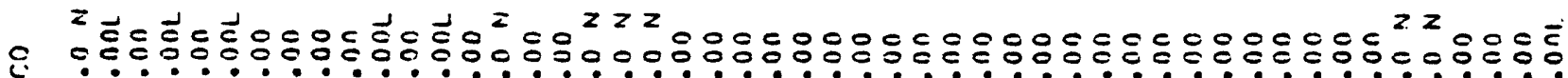

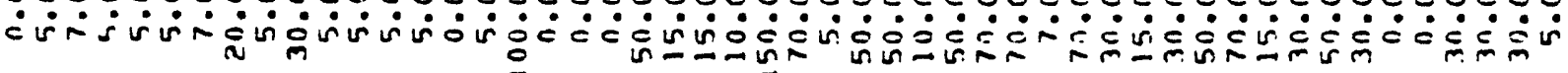

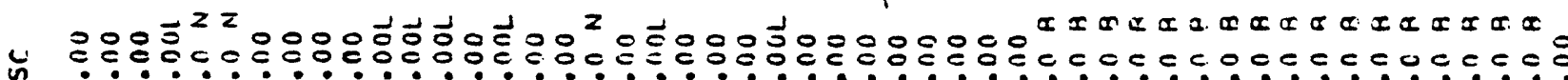

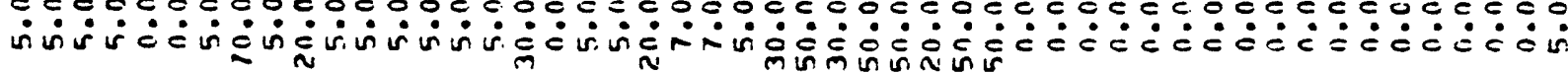

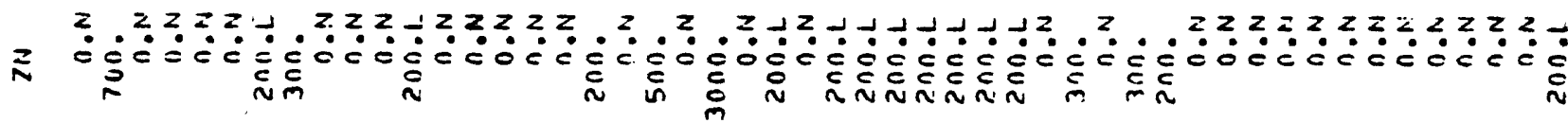

」

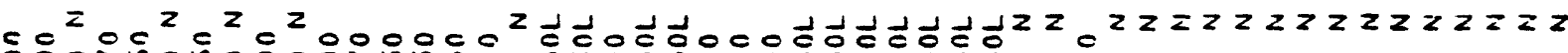

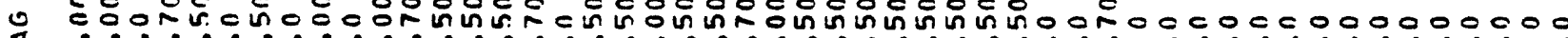

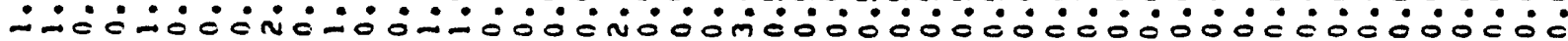

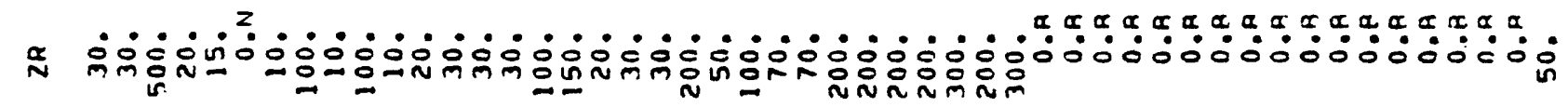

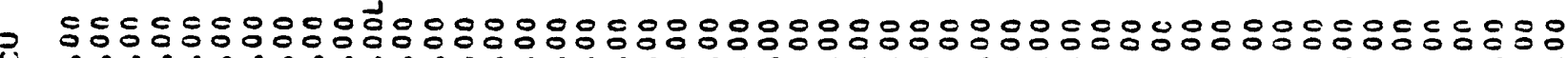

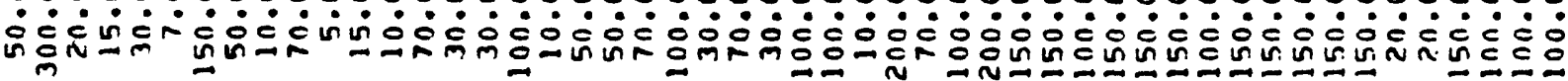

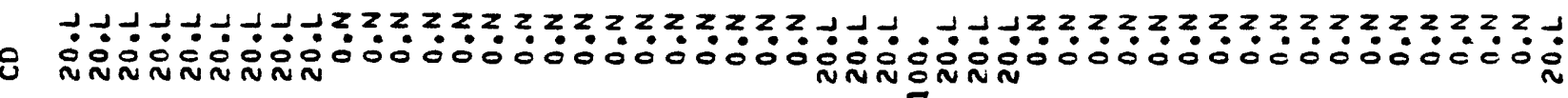

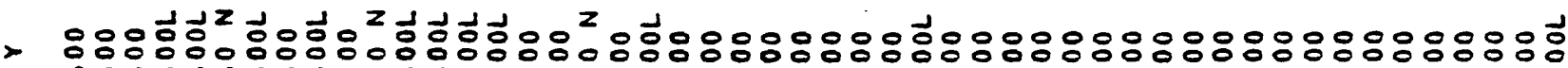

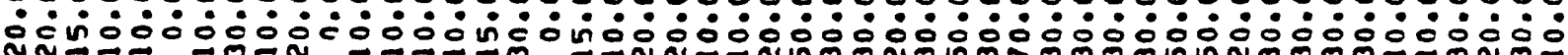




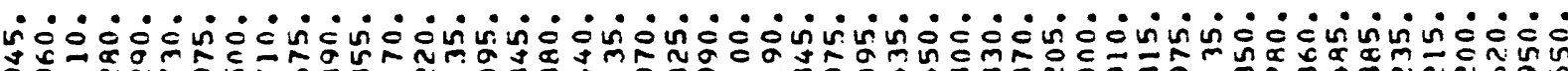

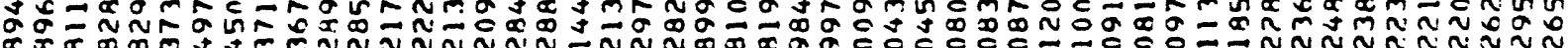

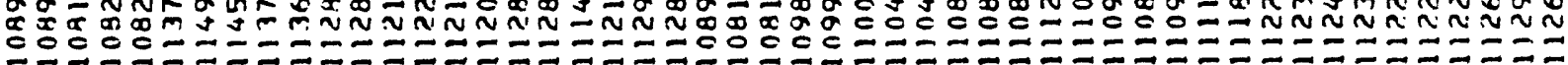

ம்

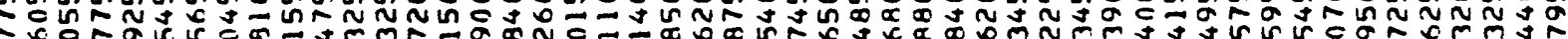
- NO

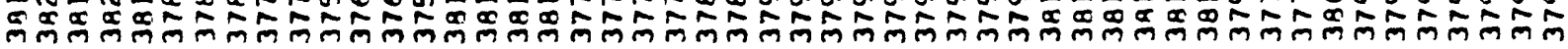

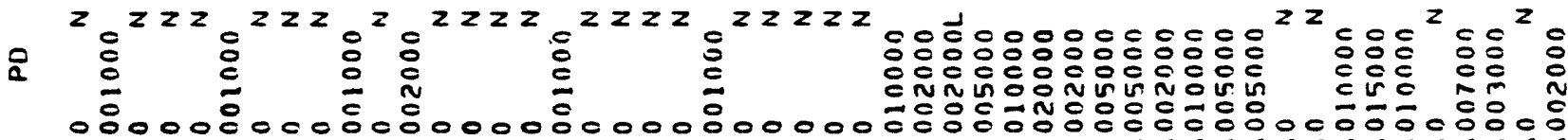
:00:00 $2 Z 2 Z 2 Z 2 Z 2 Z 2 Z 2 Z 2 Z 2 Z 2 Z 2 Z 2 Z 2 Z 2$ a<smiles>CCO[As]</smiles>

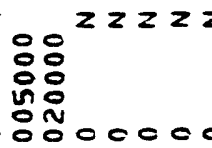

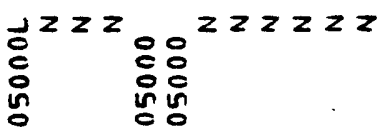
00000000000000000000000000000000000000000000000000

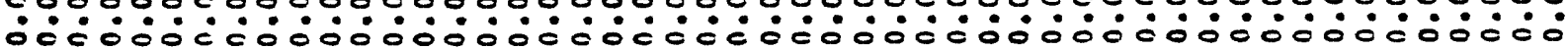

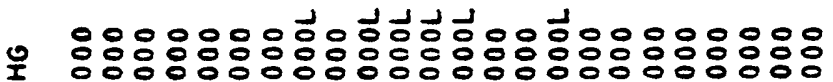

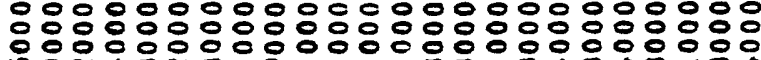

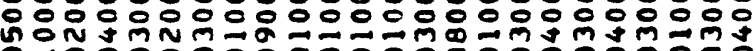

20000000000000000000000000

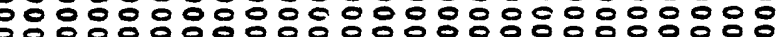
응영영영영영영영영영영영영

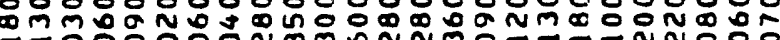

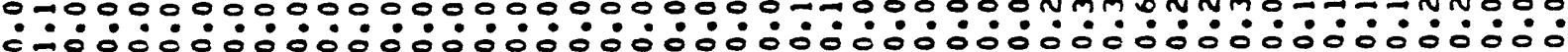




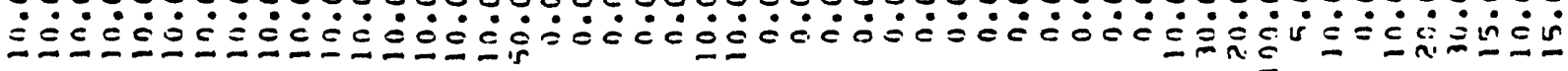

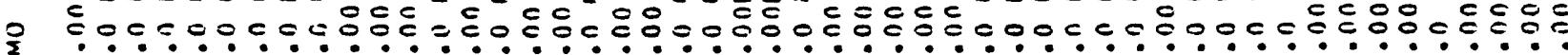

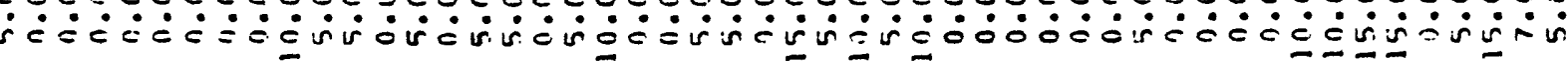

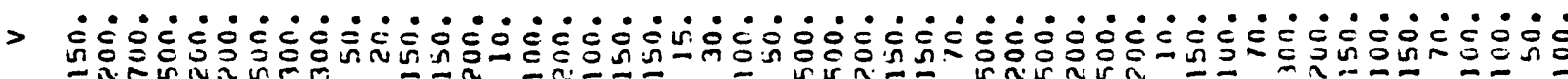

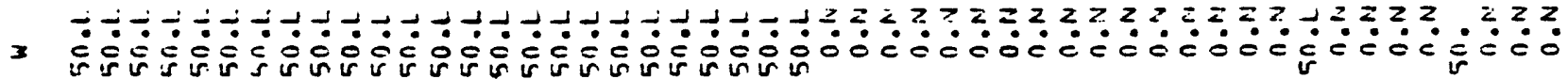

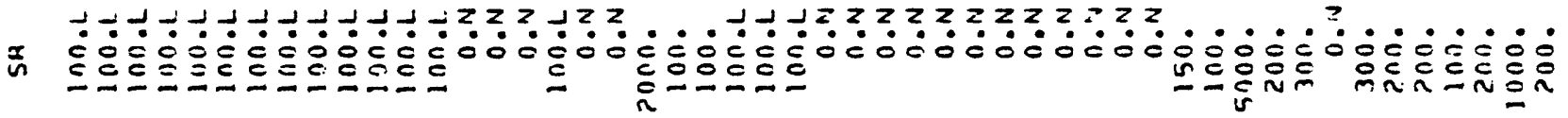

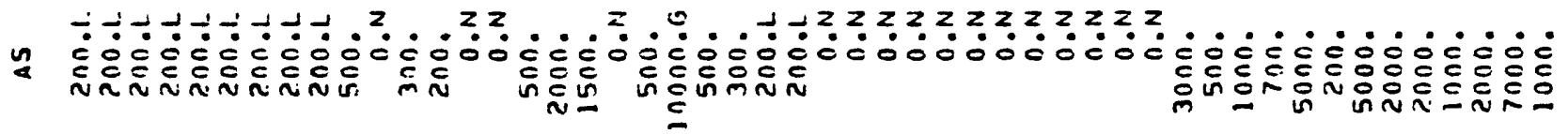

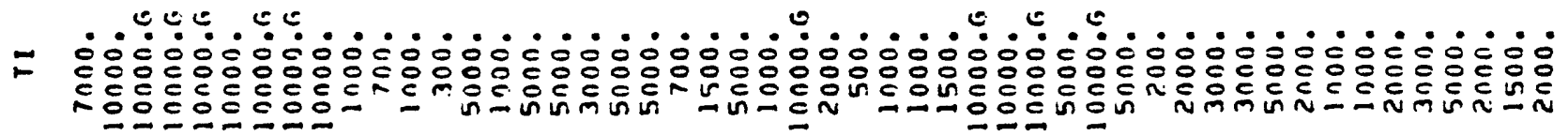

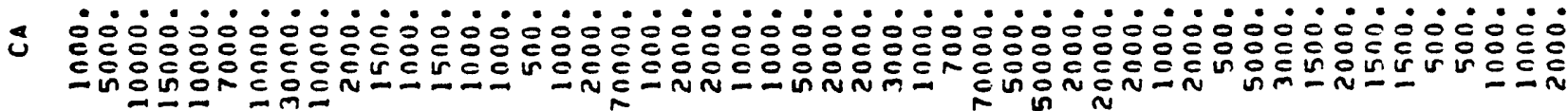

u

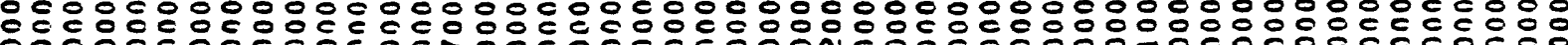

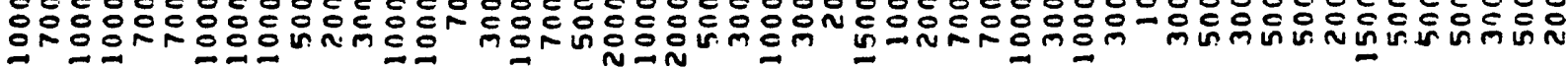

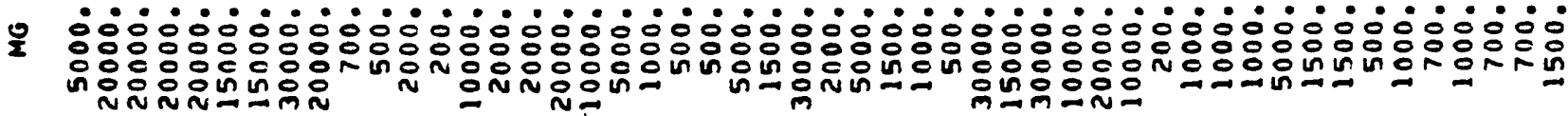


它

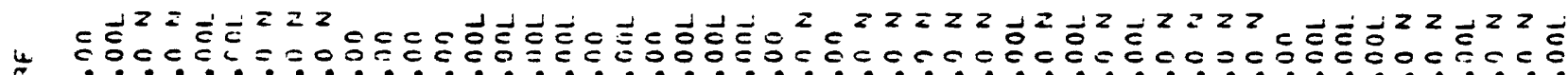

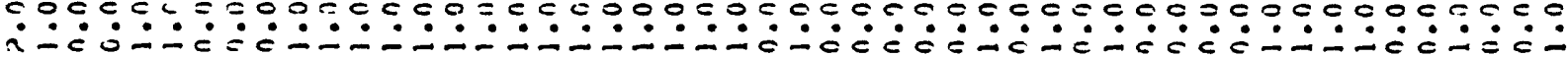

r

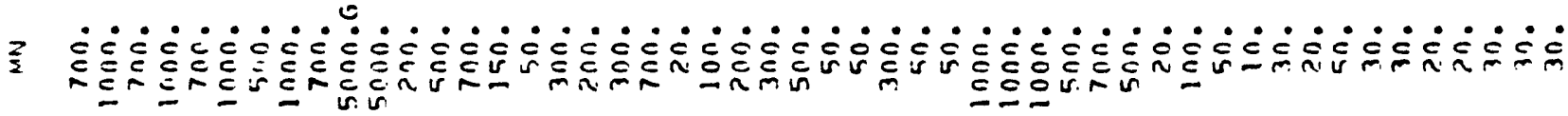

z

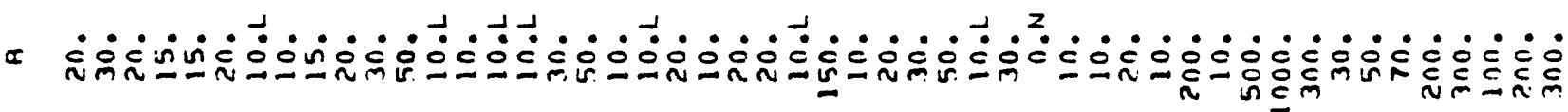

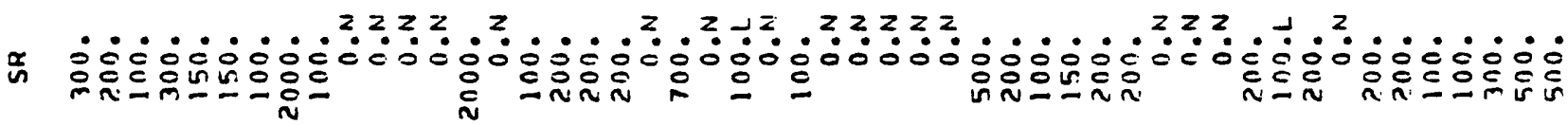

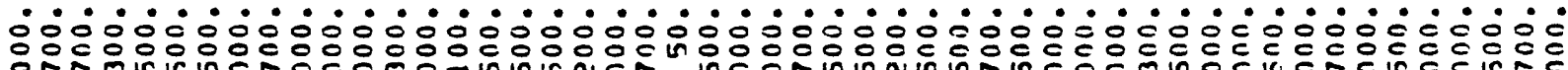

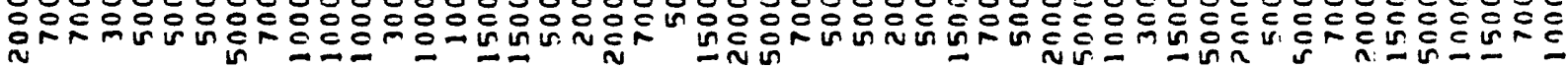

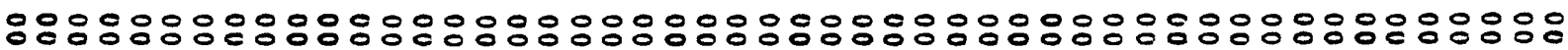

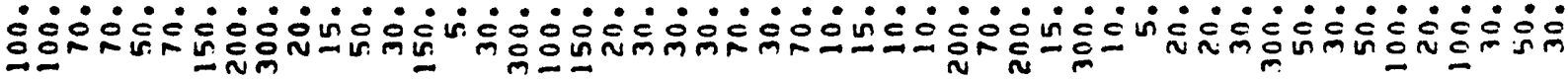

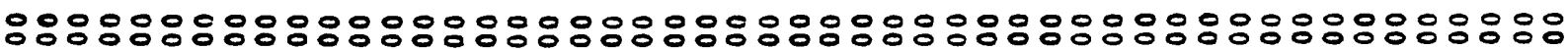

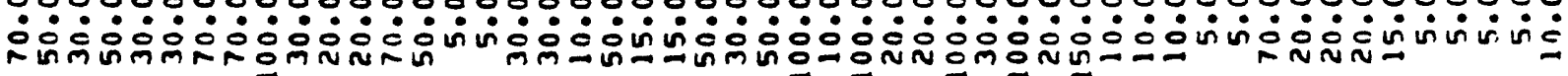




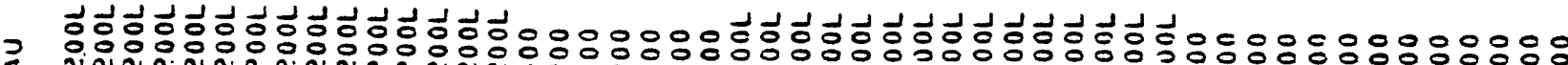

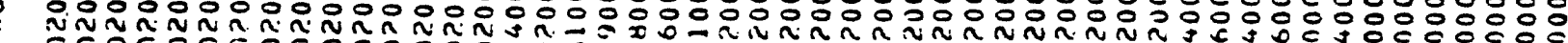

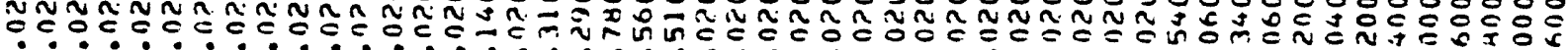

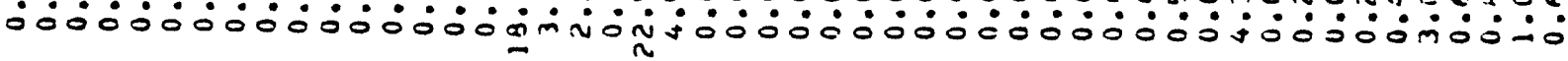

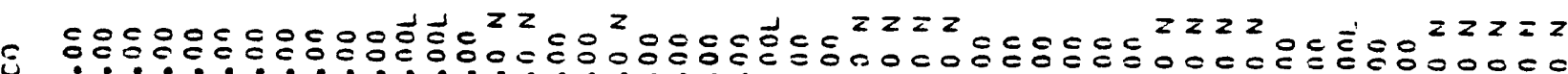

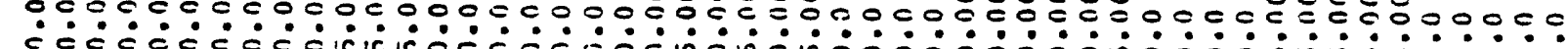

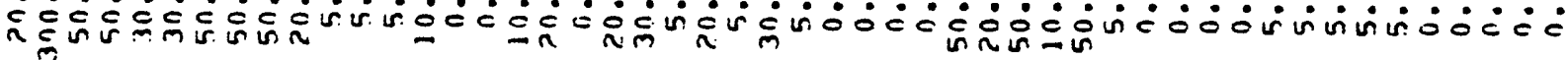

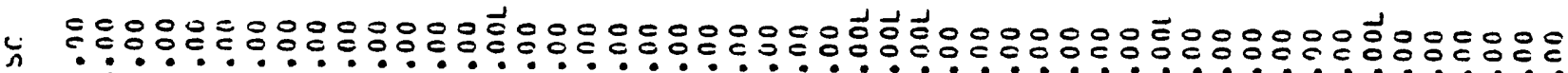

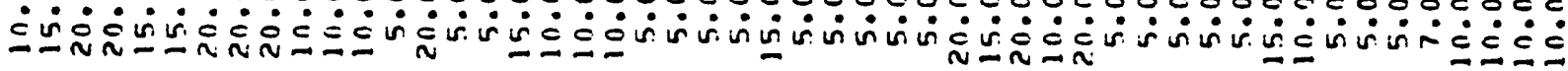

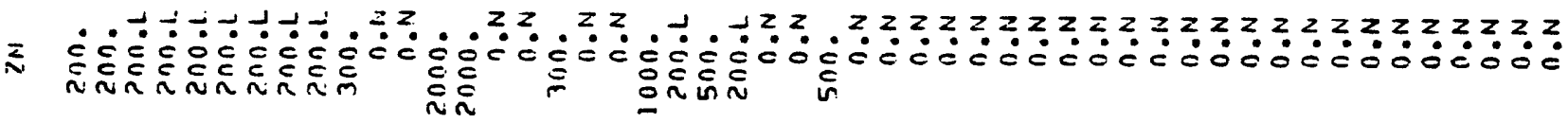

-

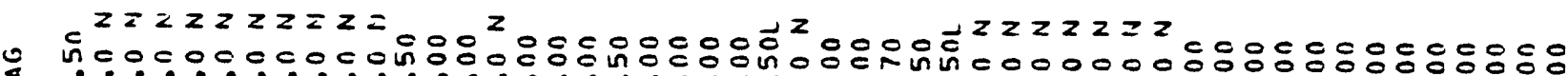

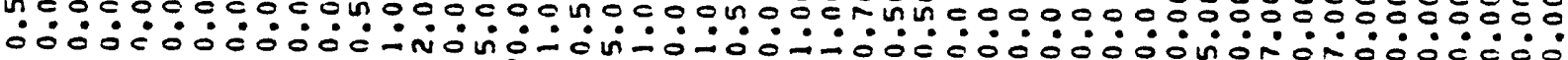

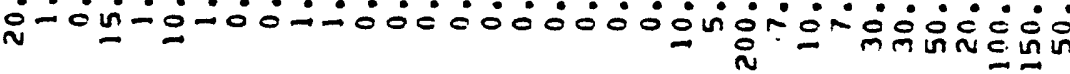

N

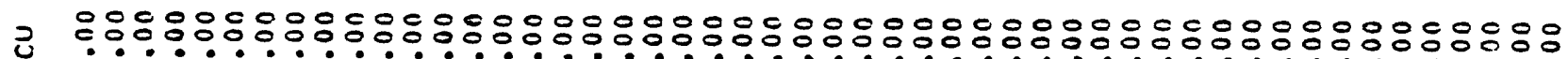

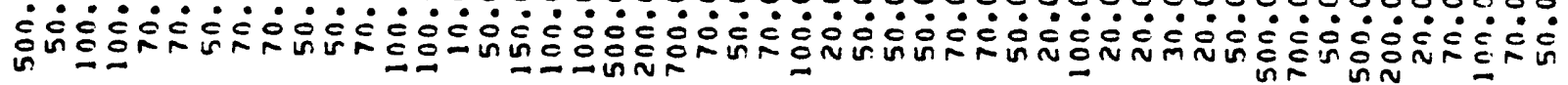

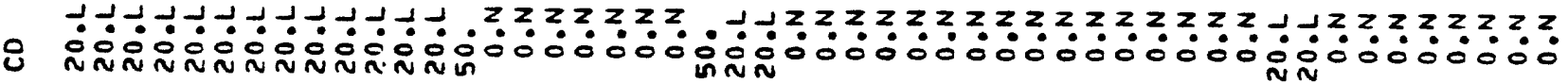

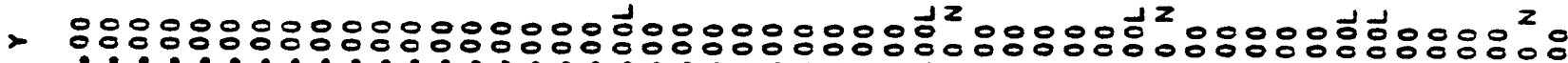

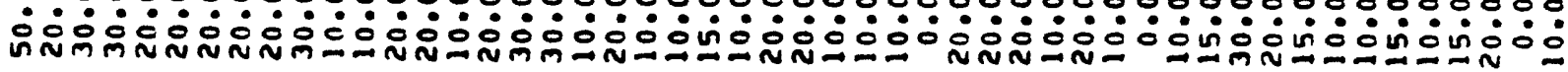

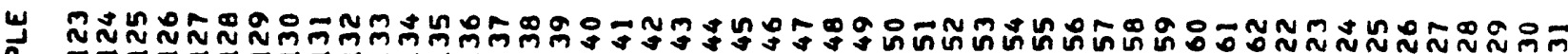

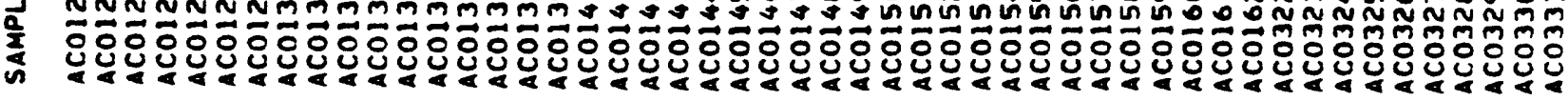


ح

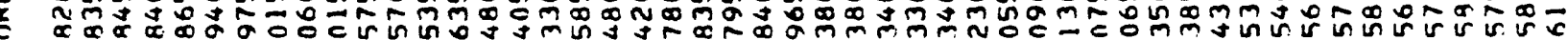

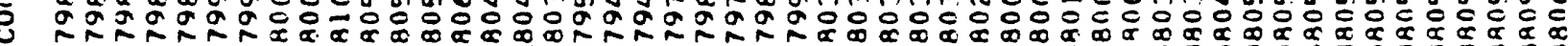

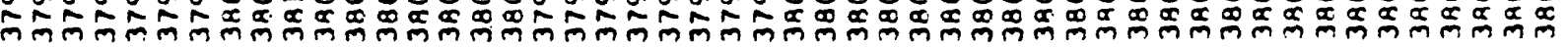

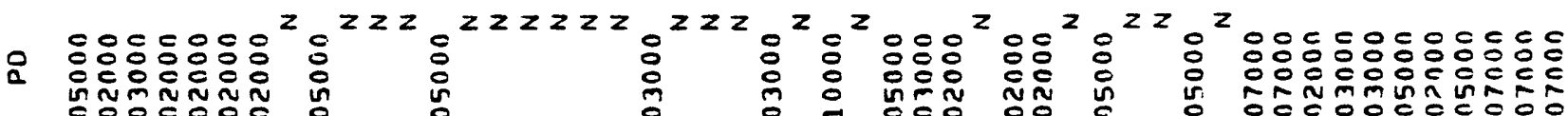

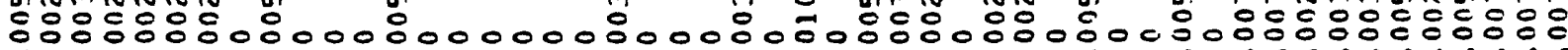

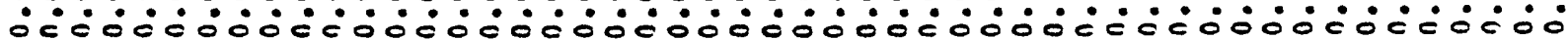

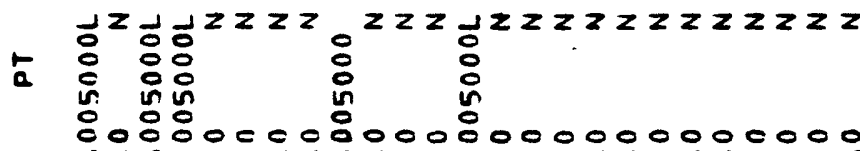<smiles>CCCC=C=C=C=[As](C)=O</smiles>

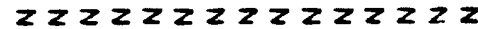
0080000000000000000000000000000000000000000000000

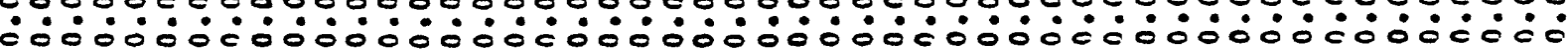

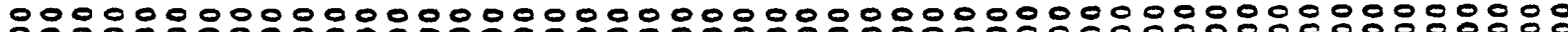

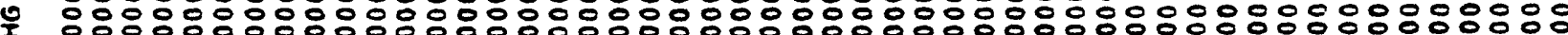

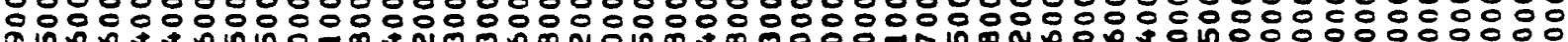
能员:

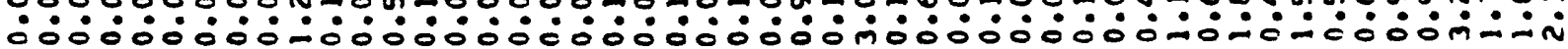




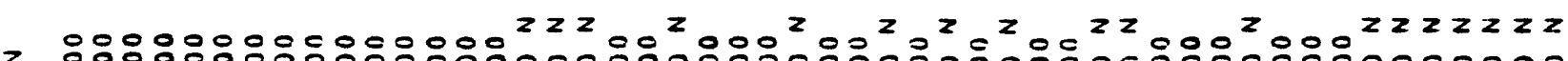

I

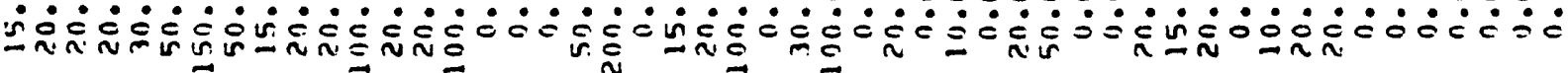

$$
\rightarrow-n-
$$

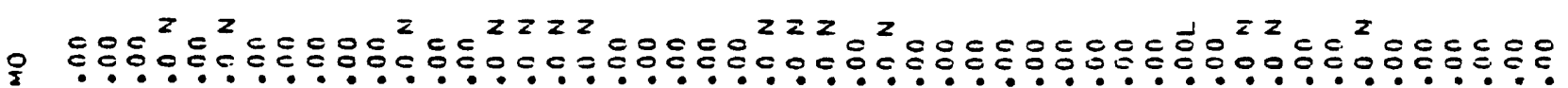

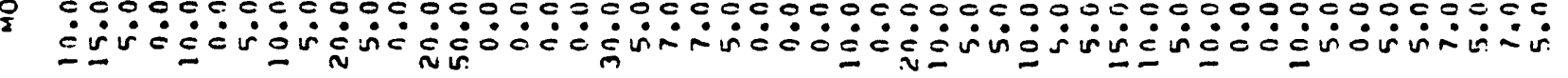

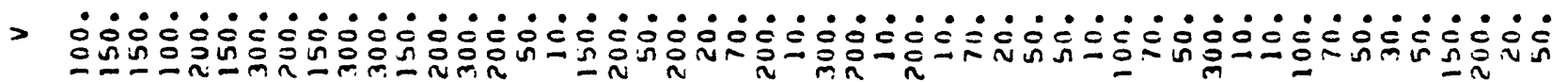

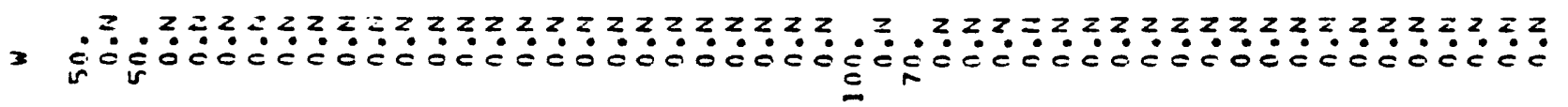

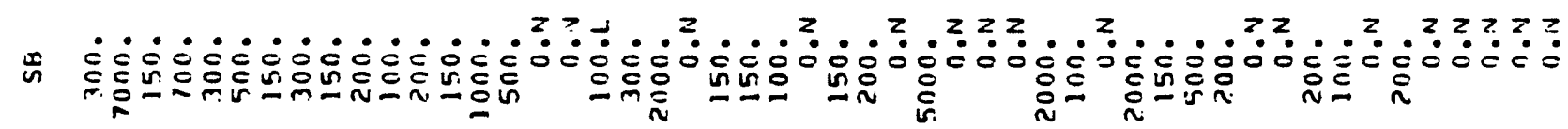

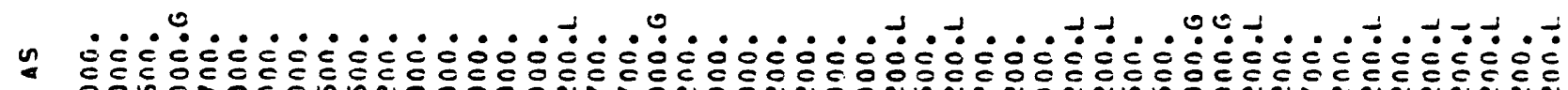

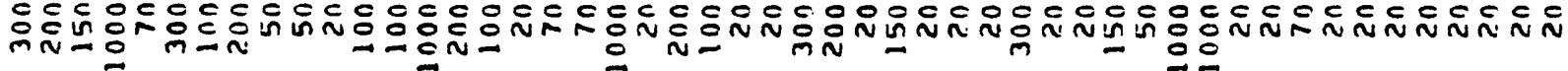

$=\quad \dot{0} \dot{0} \dot{0} \dot{0} \dot{0} \dot{0} \dot{0} \dot{0} \dot{0} \dot{0} \dot{0} \dot{0} \dot{0} \dot{0} \dot{0} \dot{0} \dot{0} \dot{0} \dot{0} \dot{0} \dot{0} \dot{0} \dot{0} \dot{0} \dot{0} \dot{0} \dot{0} \dot{0} \dot{0} \dot{0} \dot{0} \dot{0} \dot{0} \dot{0} \dot{0} \dot{0} \dot{0}$

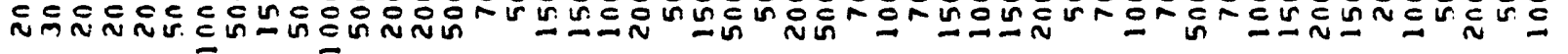

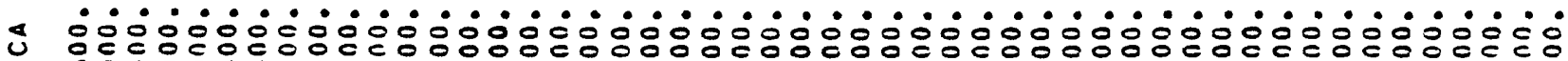
nE은에

$\omega$ L

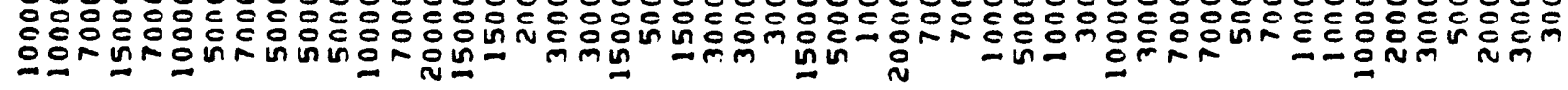

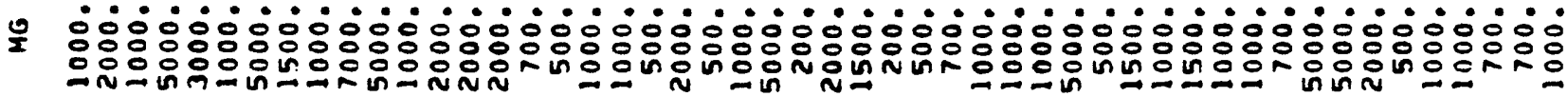




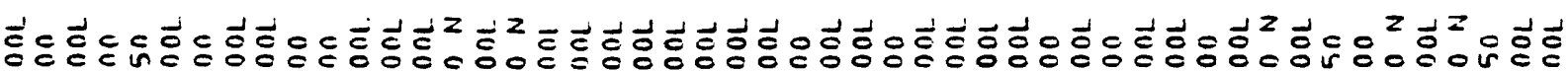
แ

×

z

a

o

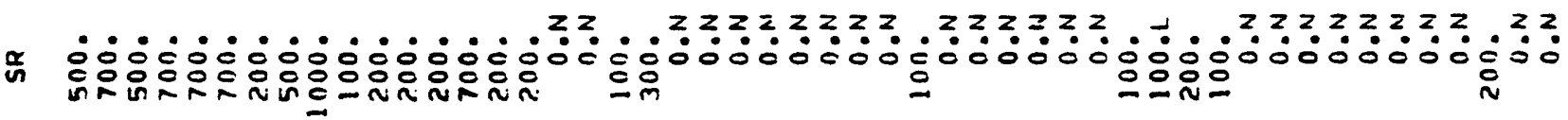

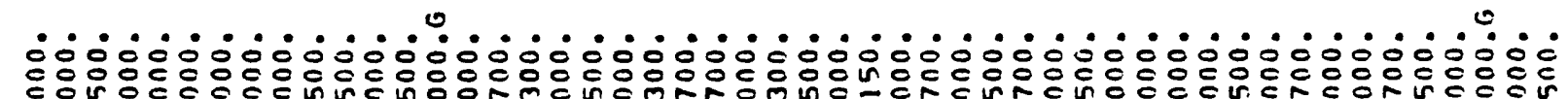

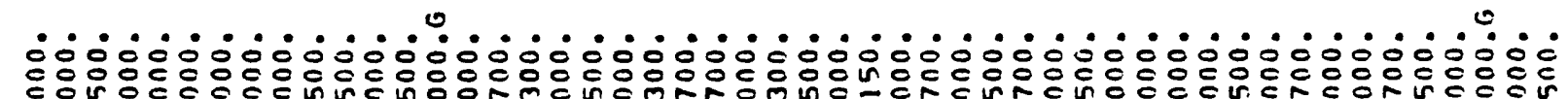

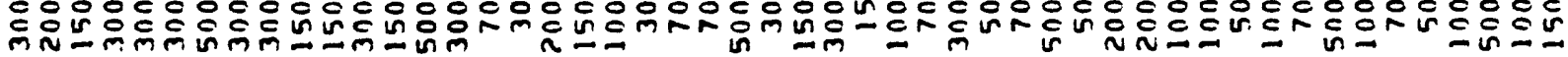

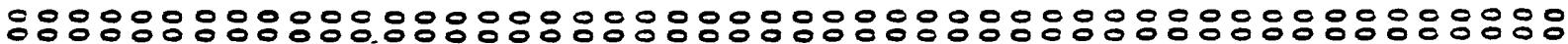
ñ

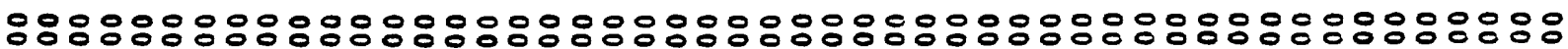

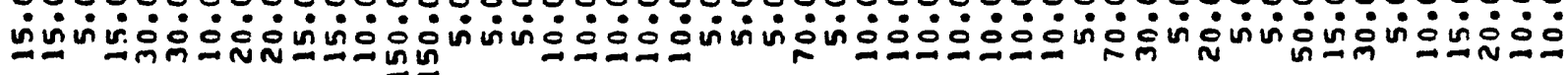




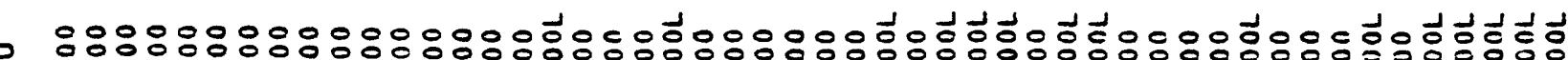

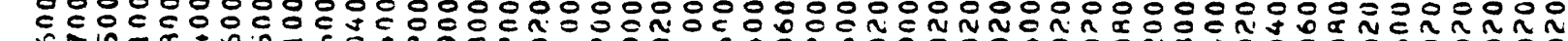

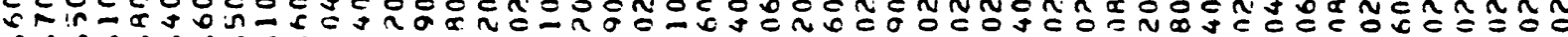

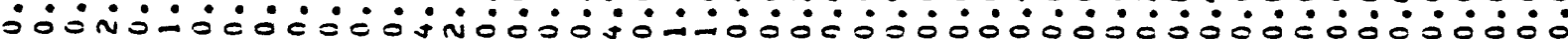

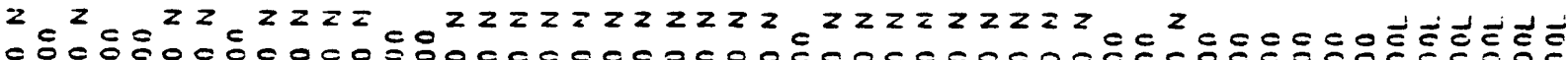

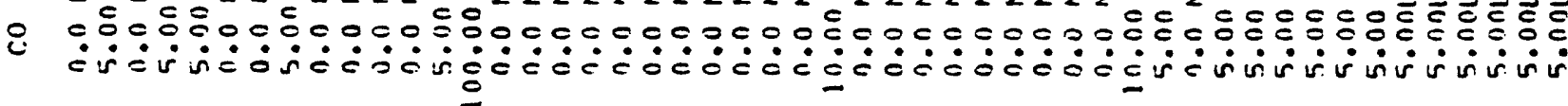

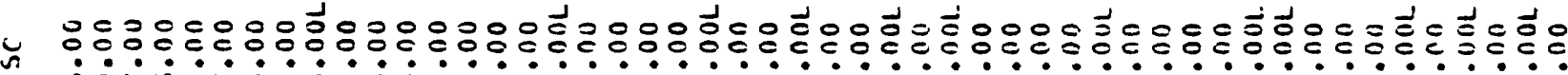

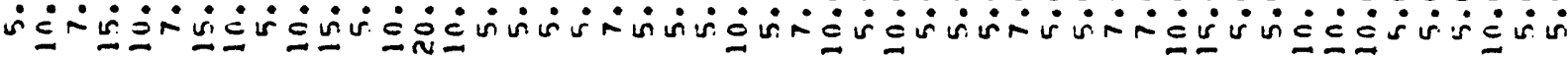

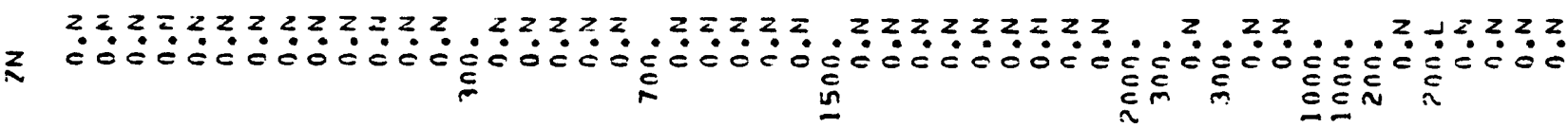

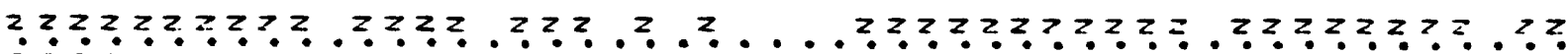

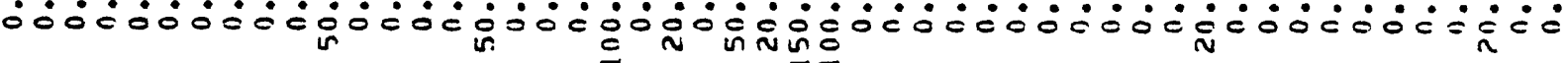

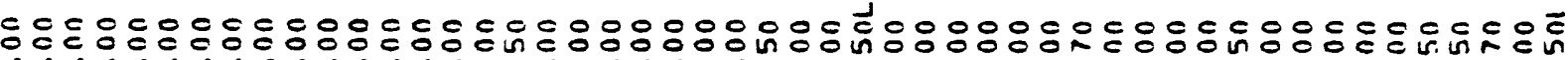

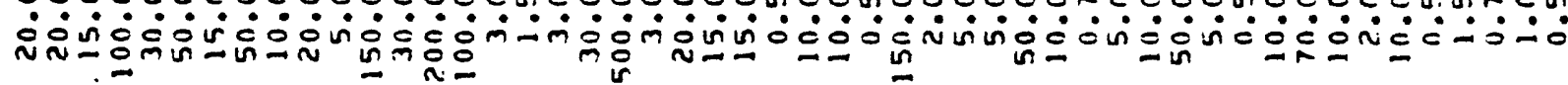

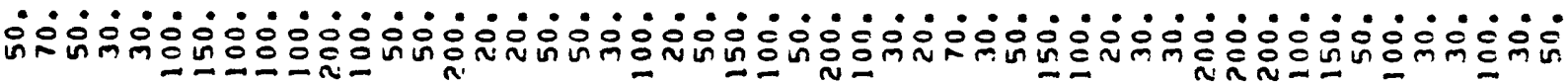

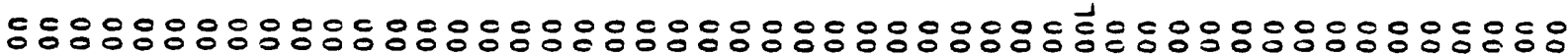

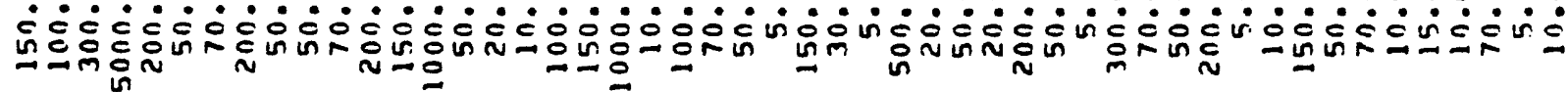

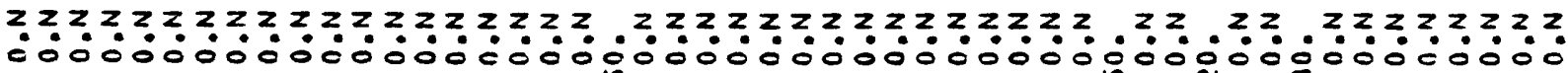
in

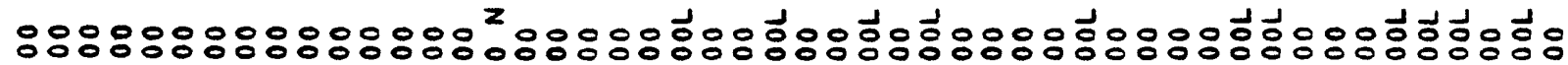 ón}




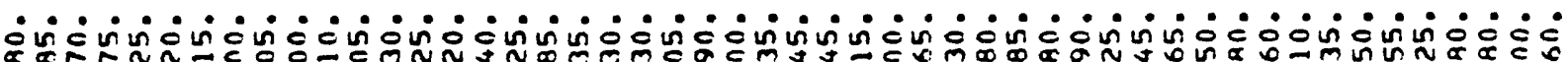

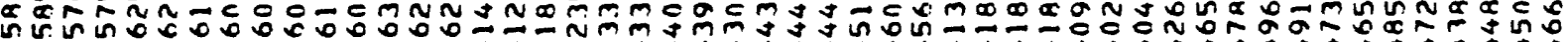

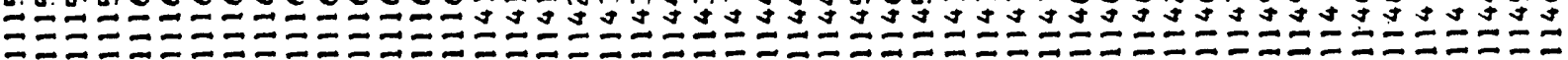

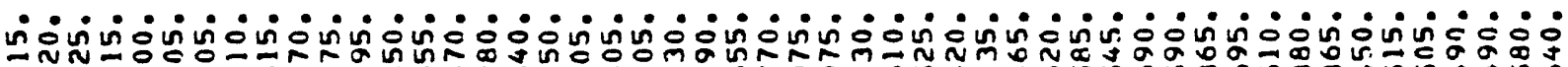
준

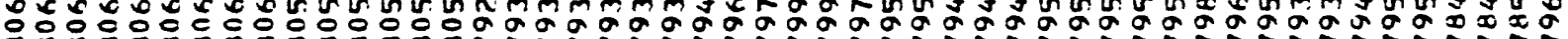

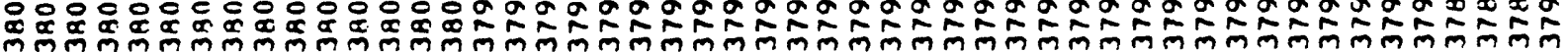

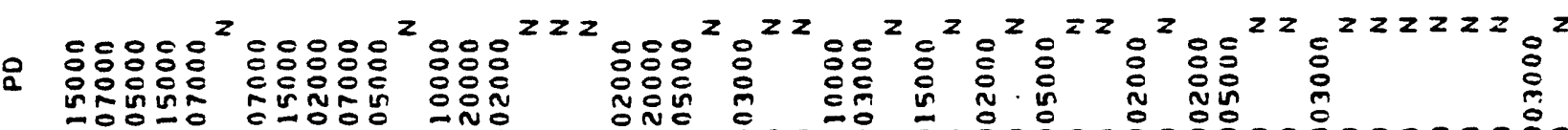

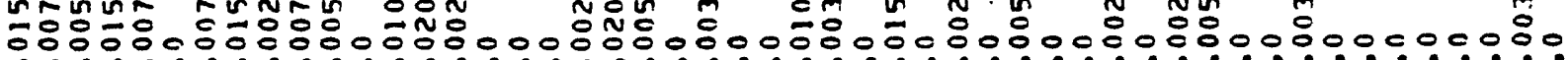

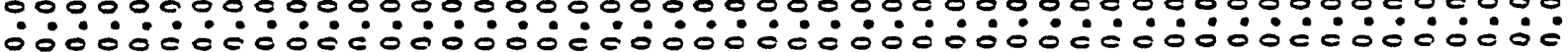

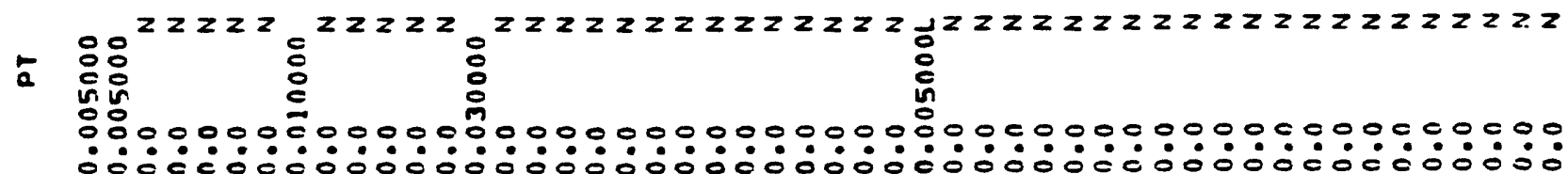

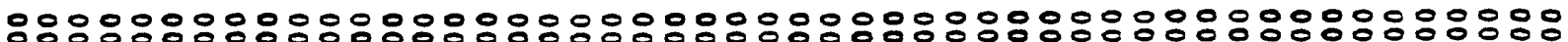

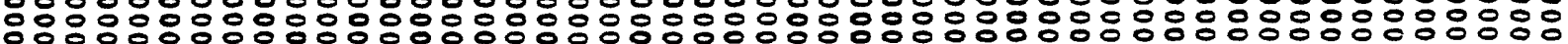

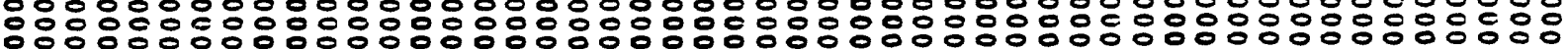

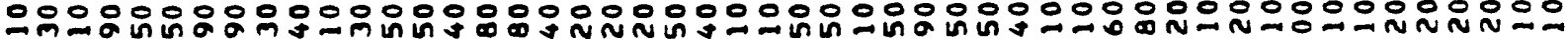

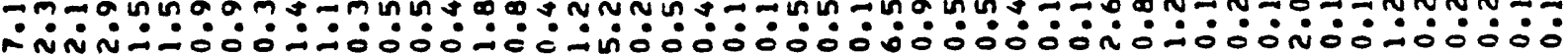




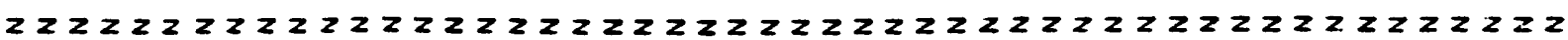

00000000000000000000000000000000000000000000000000

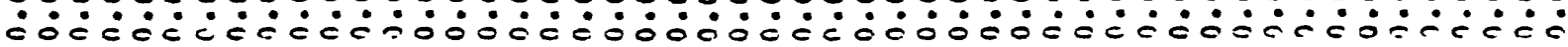

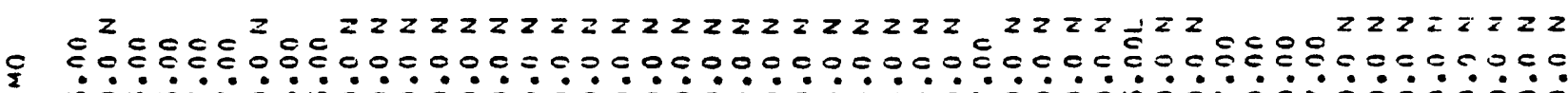

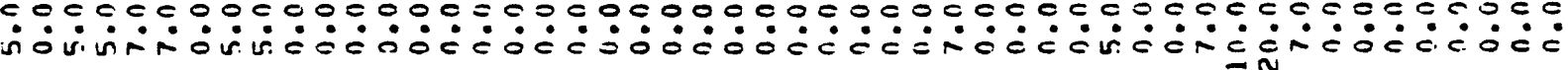

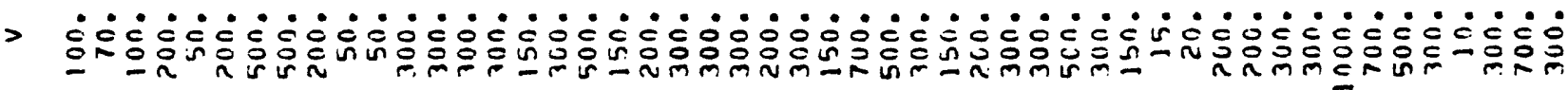

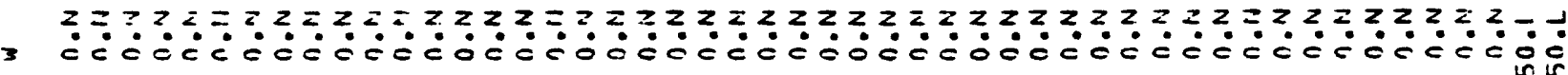

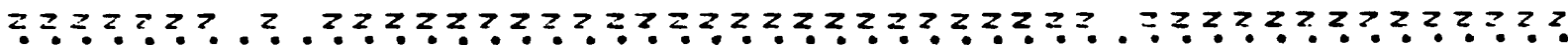

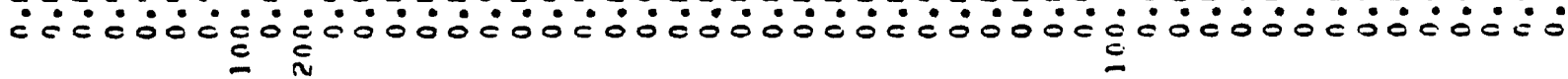

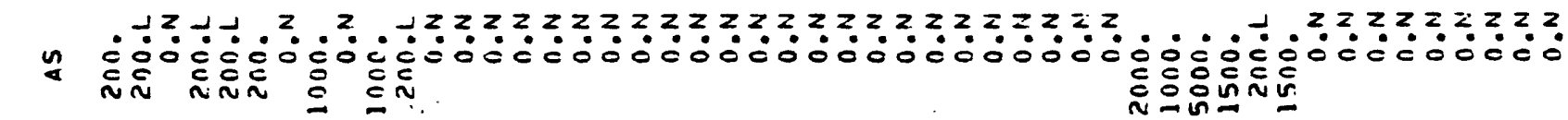
NN nNa 5 . 응ํำ

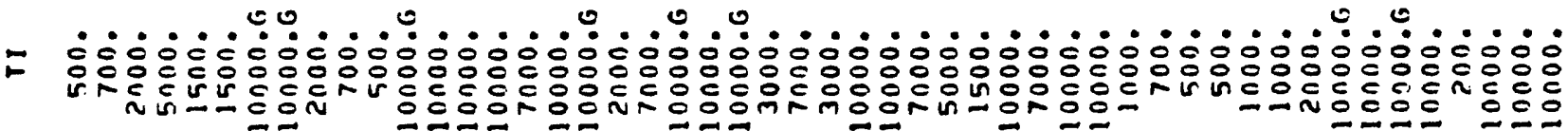

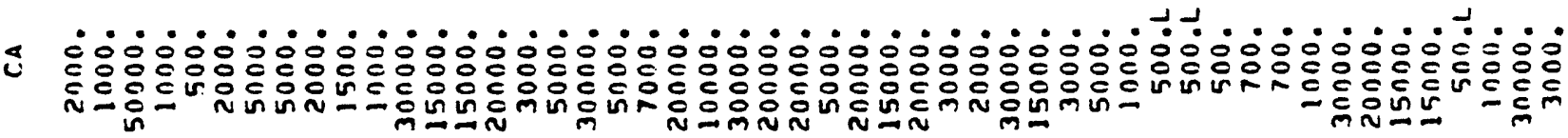

u

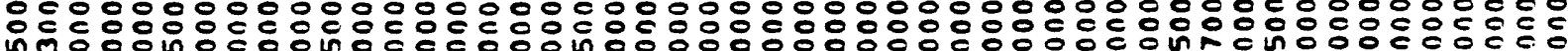

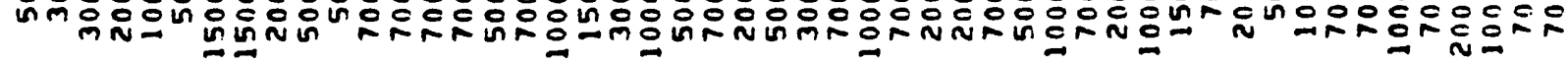

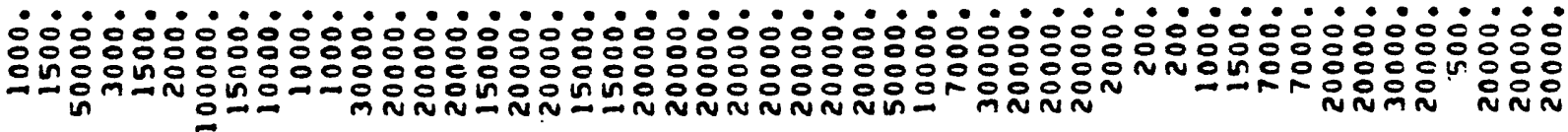




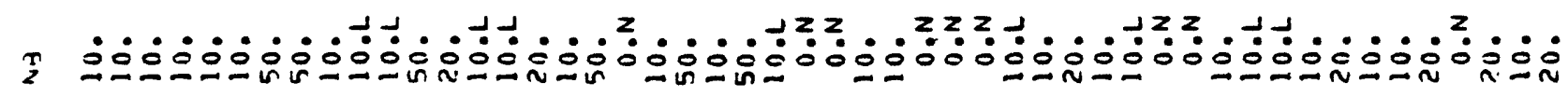

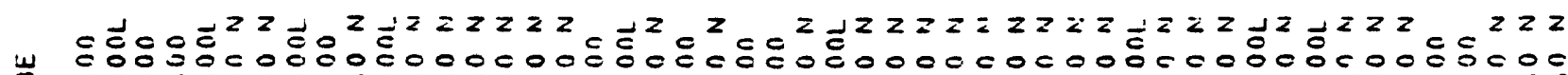
w

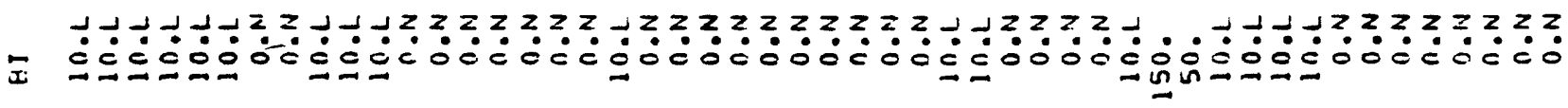

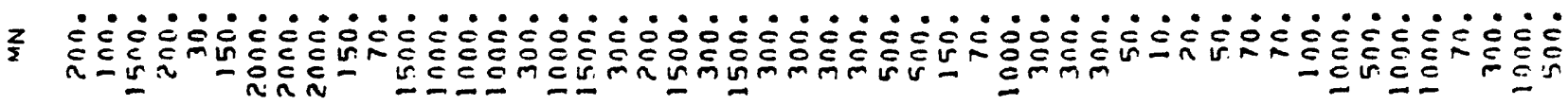

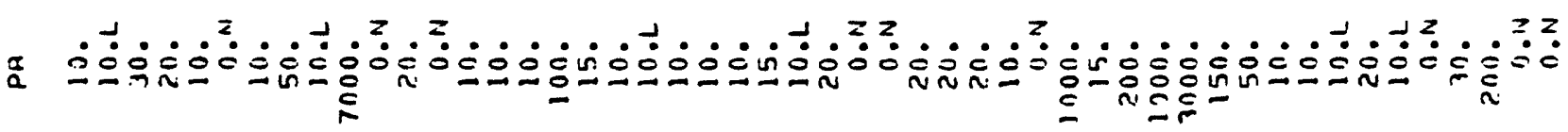

-

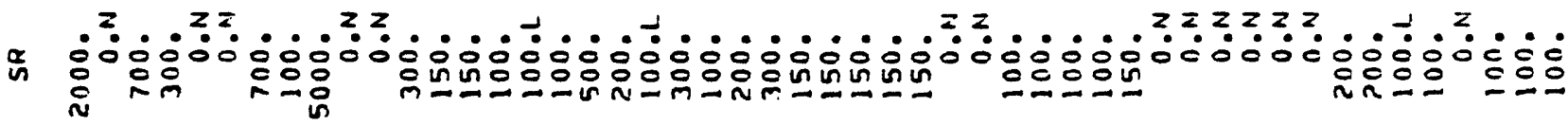

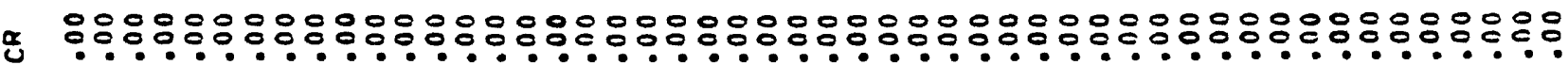

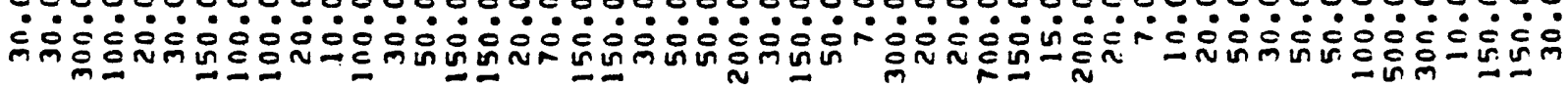

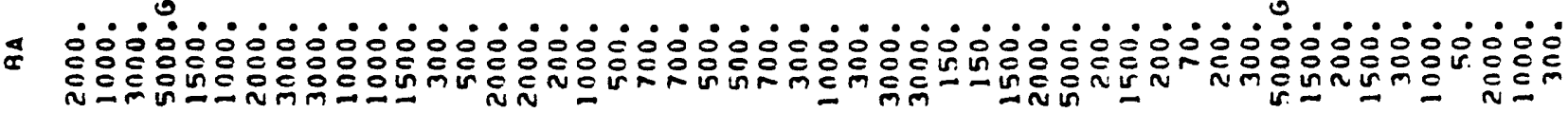

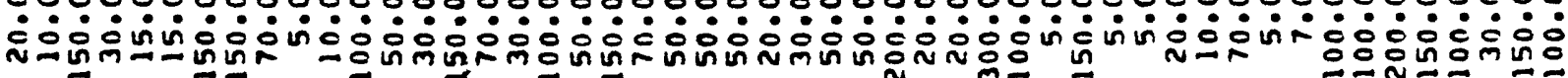




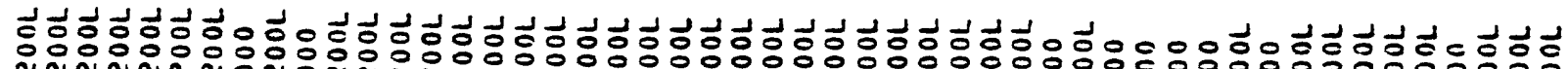

Z NิNĩNiñ

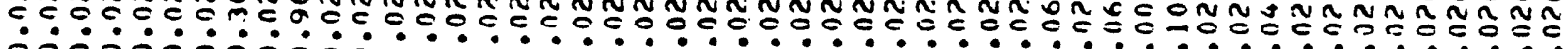

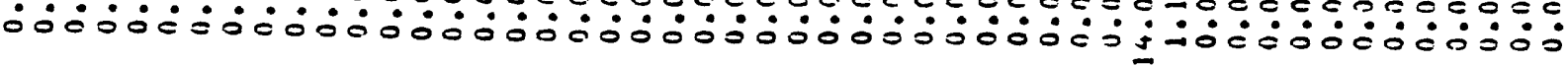

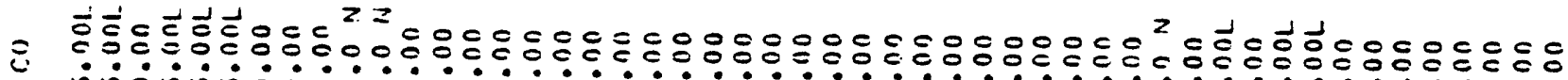

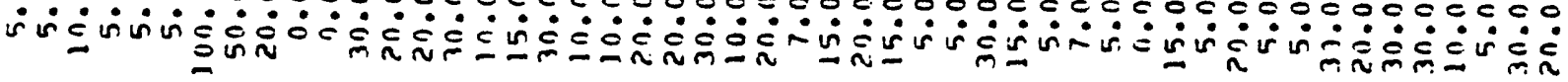

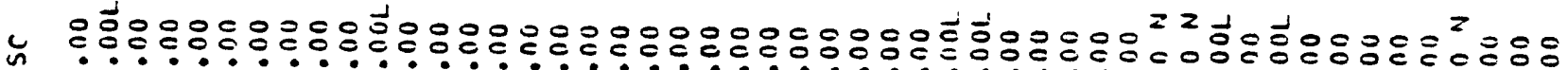

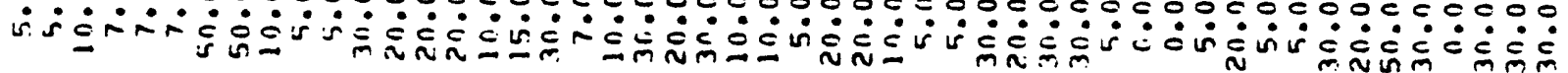

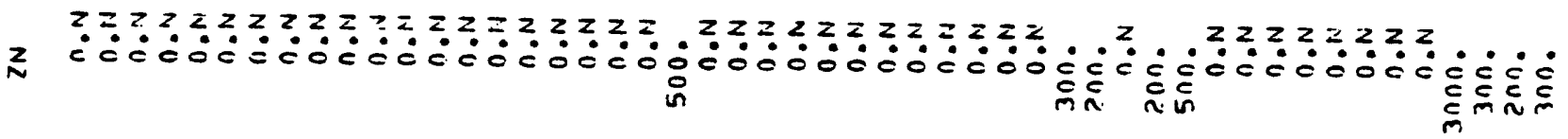

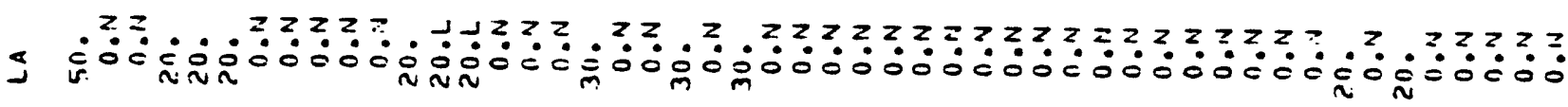

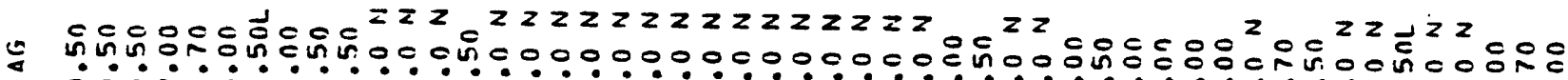

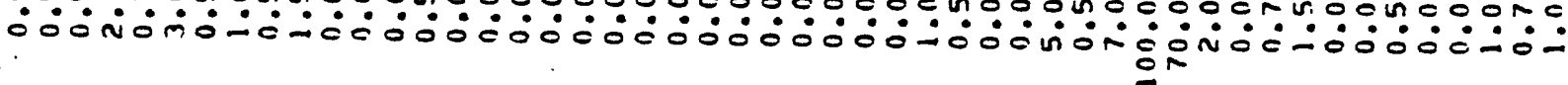

N

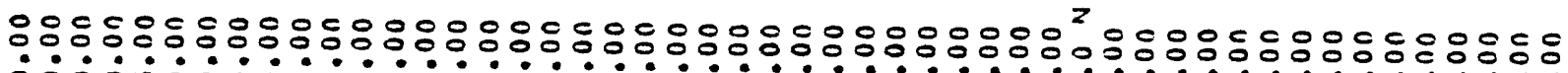

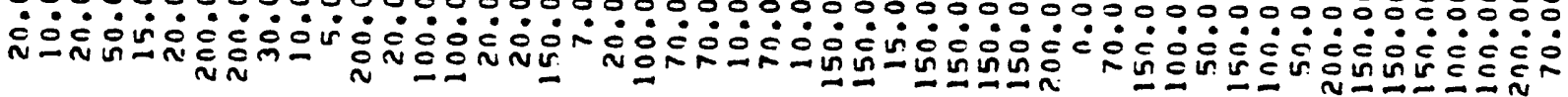

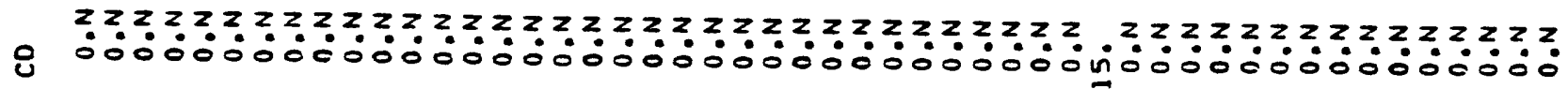

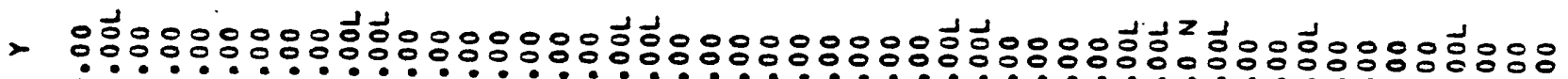

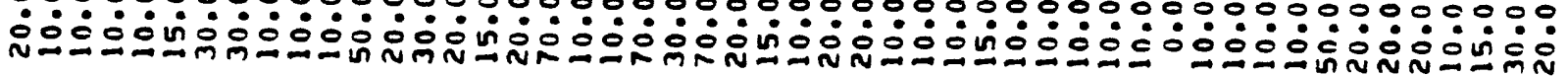




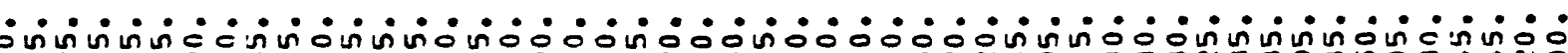

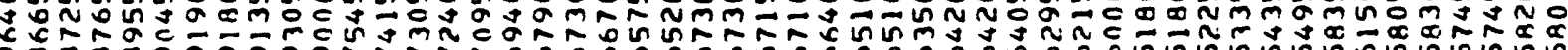

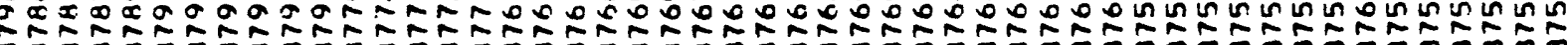

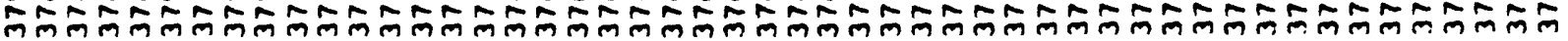

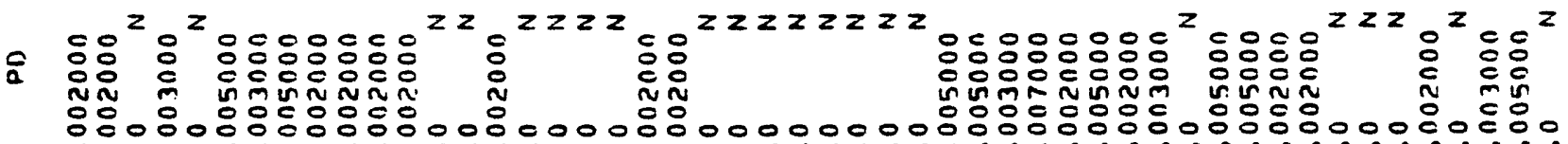

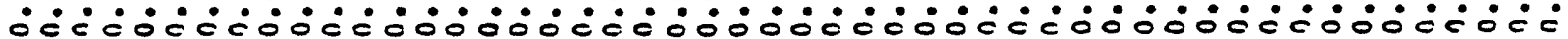

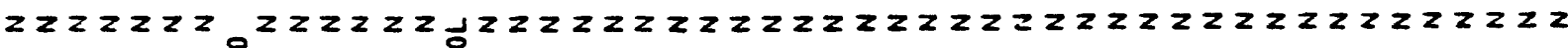

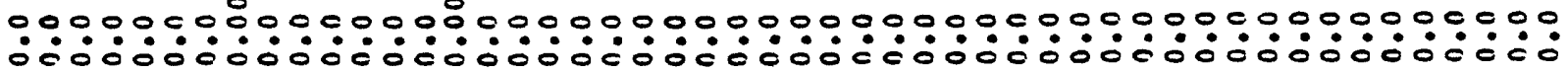

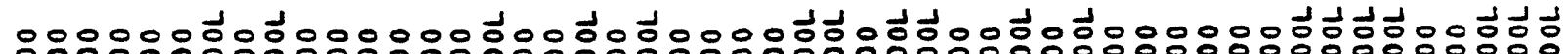

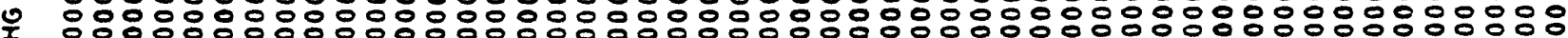

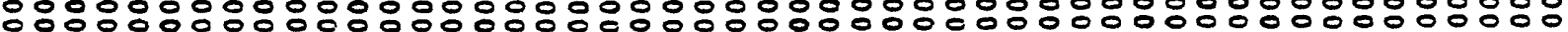

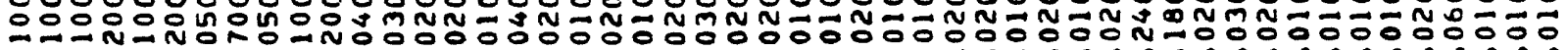
$\therefore 000000010000000000000000000000000000000000000000$ 


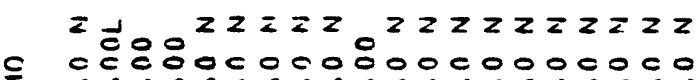

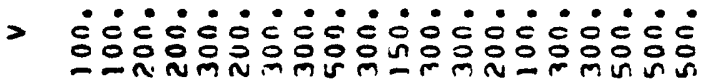

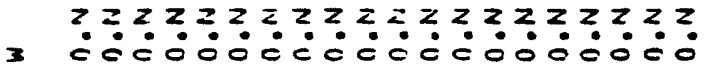

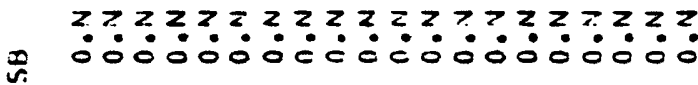

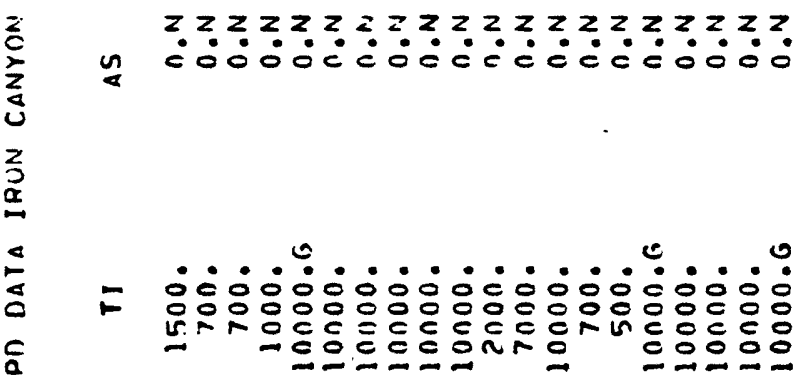

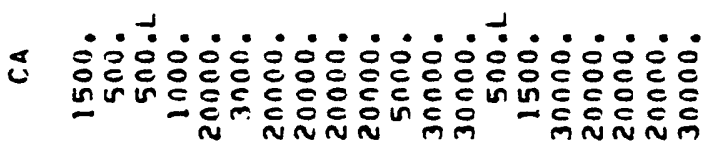

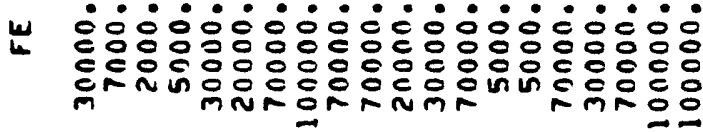

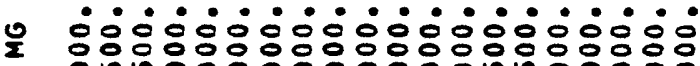

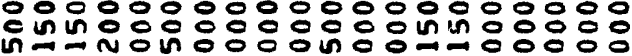

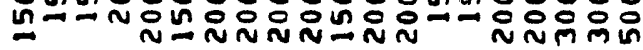




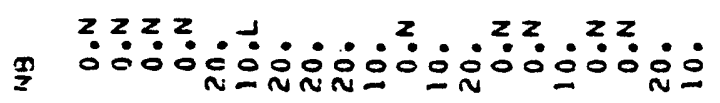

$\stackrel{5}{\vdots}$

2ZZZZZZZZZZZZZZZ2ZZ

w

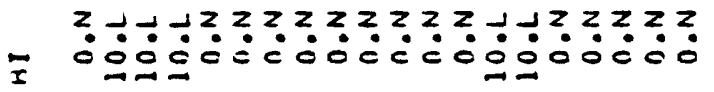

z

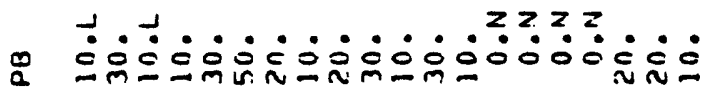

3
3
3
3
3
$\vdots$
0
$\vdots$
0
0
0
0
0

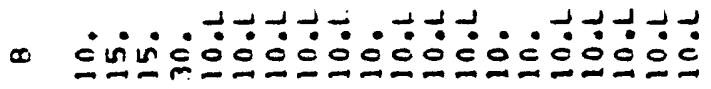

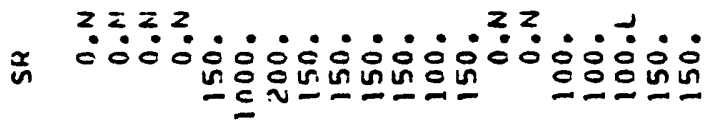

×

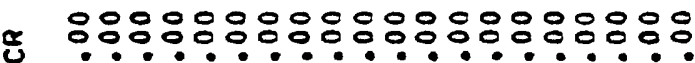

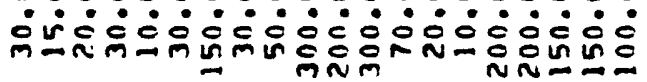

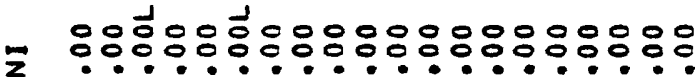
¿ல்தn்

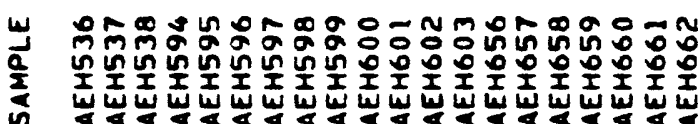




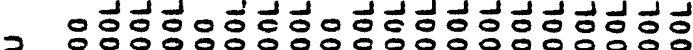

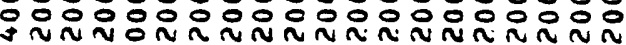

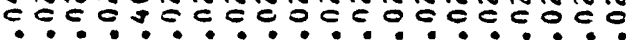

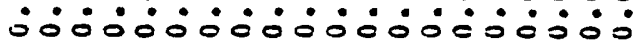

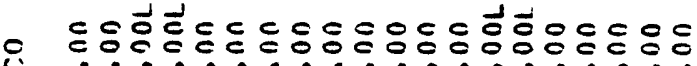

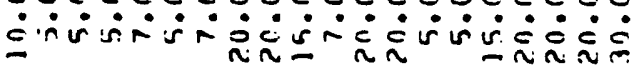

u

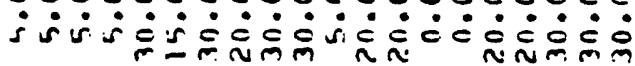

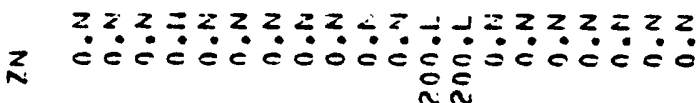

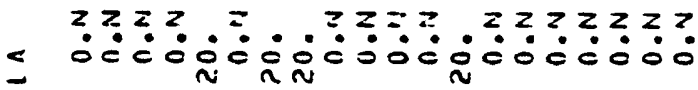

3
0
2
0
0
2
0
3
$\vdots$
5
0
0
0
1
$\frac{1}{a}$

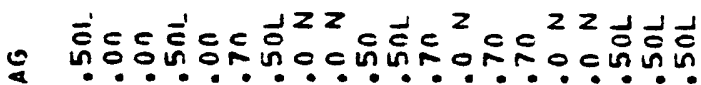

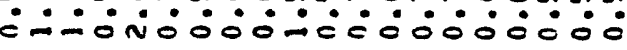

N

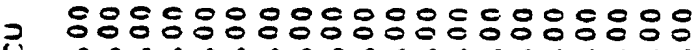

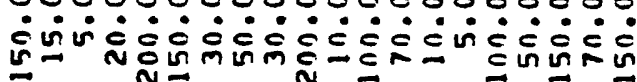

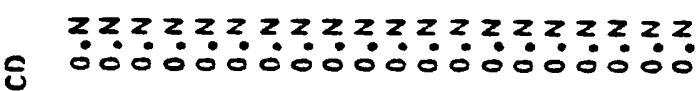

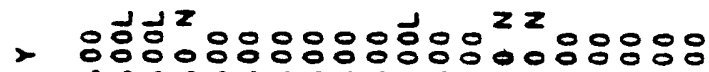

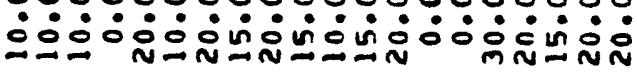

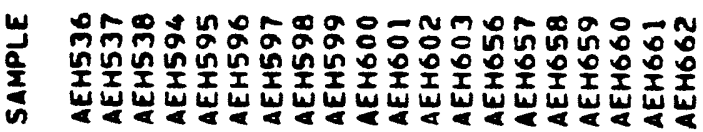




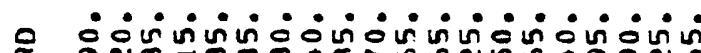

\&

C nin

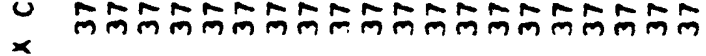

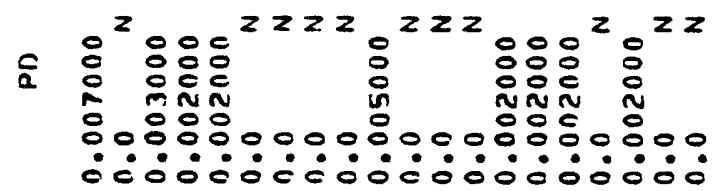
a

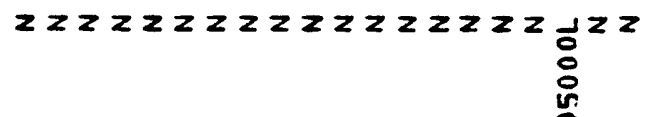

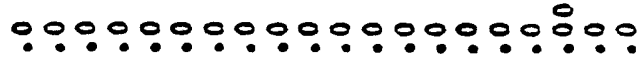

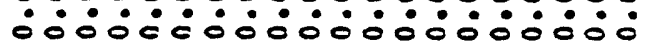

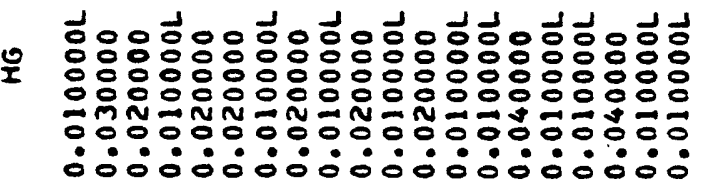

